\title{
NUMERICAL SOLUTION OF NONLINEAR ELASTICITY PROBLEMS WITH LAVRENTIEV PHENOMENON
}

\author{
YU BAI \& ZHIPING LI ${ }^{\dagger}$ \\ LMAM \& SCHOOL OF MATHEMATICAL SCIENCES, \\ PEKING UNIVERSITY, BEIJING 100871, P.R.CHINA
}

\begin{abstract}
A convergence theory is established for a truncation method in solving polyconvex elasticity problems involving the Lavrentiev phenomenon. Numerical results on a recent example by Foss et al, which has a polyconvex integrand and admits continuous singular minimizers, not only verify our convergence theorems but also provid a sharper estimate on the upper bound of a perturbation parameter for the existence of the Lavrentiev phenomenon in the example.
\end{abstract}

\section{InTRODUCTION}

Let $\Omega \subset R^{n}$ be an open and bounded set. Let $f: \Omega \times R^{m} \times R^{m n} \rightarrow \bar{R}=$ $R \cup\{+\infty\}$ be given. Then, the fundamental problem of the calculus of variations can be informally described as minimizing the functional $I: W^{1,1}\left(\Omega ; R^{m}\right) \rightarrow \bar{R}$, defined by

$$
I(\mathbf{u})=\int_{\Omega} f(\mathbf{x}, \mathbf{u}(\mathbf{x}), D \mathbf{u}(\mathbf{x})) d \mathbf{x}
$$

over an admissible set of functions $\mathcal{A}^{p}=\mathcal{A} \cap W^{1, p}\left(\Omega ; R^{m}\right)(p \in[1,+\infty])$ with $\mathcal{A}$ being a prescribed subset of $W^{1,1}\left(\Omega ; R^{m}\right)$. It is clear that the infimum $\inf _{\mathbf{u} \in \mathcal{A}^{p}} I(\mathbf{u})$ is non-decreasing with respect to $p$. For many classical problems, the infimum above is not affected by the value of $p$ [13]. However, consider the example given

2000 Mathematics Subject Classification. 65K10,65N30,49A10.

Key words and phrases. truncation method, Lavrentiev phenomenon, polyconvex, convergence, nonlinear elasticity.

The research was supported in part by the Special Funds for Major State Basic Research Projects (2005CB321701), NSFC (10431050, 10571006 and 10528102) and RFDP of China.

$\dagger$ Corresponding author: Zhiping Li, email address: lizp@math.pku.edu.cn. 
by Manià [21], of minimizing the integral functional

$$
I(u)=\int_{0}^{1}\left(u^{3}-x\right)^{2}\left(u^{\prime}\right)^{6} d x
$$

in the admissible functions $\mathcal{A}=\left\{u \in W^{1,1}(0,1): u(0)=0, u(1)=1\right\}$. We have

(i) The Lavrentiev phenomenon, that is

$$
\inf _{u \in \mathcal{A}^{\infty}} I(u)>\inf _{u \in \mathcal{A}^{1}} I(u)
$$

The occurrence of such a phenomenon was first discovered by Lavrentiev for a different example in 1926 [15].

(ii) If $\left\{u_{j}\right\} \subset \mathcal{A}^{p}$, for some $p \geqslant \frac{3}{2}$ and $u_{j} \rightarrow \hat{u}$ a.e. $x \in(0,1)$, where $\hat{u}=x^{\frac{1}{3}}$ is the absolute minimizer of $I$ in $\mathcal{A}^{1}$, then [5]

$$
\lim _{j \rightarrow+\infty} I\left(u_{j}\right)=+\infty
$$

This is the so called repulsion property, which is commonly seen in the problems exhibiting the Lavrentiev phenomenon.

Properties (i) and (ii) suggest that the standard finite element methods can neither detect the absolute minimizer nor determine the minimum value. Various numerical methods for detecting singular minimizers have been developed in recent years $[2,6,16,17,18,19]$ (see [7] for a survey and more references), and the corresponding convergence theorems were proved for the case when the integrand $f$ is convex with respect to the deformation gradient $D \mathbf{u}$.

It is of great practical interests that the phenomena also exist in nonlinear elasticity problems. In fact, it has long been known that discontinuous equilibrium solutions exhibit the Lavrentiev phenomenon [3]. Recently, Foss et. al. [13] gave examples of a family of nonlinear elasticity problems which have continuous singular minimizers exhibiting the Lavrentiev phenomenon. In their examples, the integrand $f$ is polyconvex with respect to the deformation gradient $D \mathbf{u}$, more precisely, it is of the form $f=f_{c}+\varepsilon f_{p}$ with $f_{c}$ convex and $f_{p}$ polyconvex, and it was shown that the Lavrentiev phenomenon exists when the parameter $\varepsilon$ is no greater than some upper bound $\varepsilon_{0}[13]$. 
In the present paper, we generalize the theory developed in [2] and establish a convergence theory for the truncation method for the case when $f$ is polyconvex, which enables reliable applications of the truncation method to polyconvex elasticity problems.

The rest of the paper is organized as follows. Some preliminary definitions and results, which are useful in the convergence analysis of the method, are given in Section 2. In Section 3, we establish the convergence theorems for the truncation method for polyconvex integrands. In Section 4, we show numerical results on the examples within the framework of two-dimensional nonlinear elasticity given by Foss [13]. They not only verify our convergence theorems but also suggest a sharper estimate on the upper bound of the perturbation parameter $\varepsilon$ for the existence of the Lavrentiev phenomenon.

\section{Preliminary DeFinitions AND RESUlts}

Let $\Omega \subset R^{n}$ be bounded and open. We first introduce some definitions required for the formulation of a lower semicontinuity theorem.

Definition 2.1. A function $g: \Omega \times R^{m} \times R^{k} \longrightarrow \bar{R}$ is called $L \otimes B$-measurable, if it is measurable with respect to the $\sigma$-algebra generated by products of measurable subsets of $\Omega$ and Borel subsets of $R^{m} \times R^{k}$.

Definition 2.2. A function $g: \Omega \times R^{m} \times R^{k} \longrightarrow R$ is called a Carathéodory function, if

(i) $g(\cdot, \mathbf{u}, \mathbf{v})$ is measurable for every $\mathbf{u} \in R^{m}, \mathbf{v} \in R^{k}$,

(ii) $g(\mathbf{x}, \cdot, \cdot)$ is continuous for almost every $\mathbf{x} \in \Omega$.

Definition 2.3. A sequence of functions $g_{M}: \Omega \times R^{m} \times R^{k} \longrightarrow \bar{R}$ is said to converge to $g: \Omega \times R^{m} \times R^{k} \longrightarrow \bar{R}$, locally uniformly in $\Omega \times R^{m} \times R^{k}$, if there exists a sequence of measurable subsets $\Omega_{l} \subset \Omega$ with meas $_{n}\left(\Omega \backslash \Omega_{l}\right) \rightarrow 0$ as $l \rightarrow \infty$ such that, for each $l \in \mathcal{N}$ and any compact subset $G \subset R^{m} \times R^{k}$,

$$
g_{M}(\mathbf{x}, \mathbf{u}, \mathbf{v}) \rightarrow g(\mathbf{x}, \mathbf{u}, \mathbf{v}) \text { unifomly on } \Omega_{l} \times G \text { as } M \rightarrow+\infty .
$$

Throughout this paper $\rightarrow$ denotes sequential weak convergence. The following theorem is a special case of a more general theorem given by $\mathrm{Li}$ in [20].

Theorem 2.1. Let $1 \leqslant p \leqslant+\infty$ and let $g: \Omega \times R^{m} \times R^{k} \longrightarrow \bar{R}$ satisfy 
(i) $g(\cdot, \cdot, \cdot)$ is a Carathéodory function,

(ii) $g(\mathbf{x}, \mathbf{u}, \cdot)$ is convex,

(iii) $g(\mathbf{x}, \mathbf{u}, \mathbf{v}) \geqslant a(\mathbf{x})$, for some $a(\cdot) \in L^{1}(\Omega)$.

Let $g_{M}: \Omega \times R^{m} \times R^{k} \longrightarrow R$ satisfy

(a) $g_{M}(\cdot, \cdot, \cdot)$ are $L \otimes B$-measurable,

(b) $g_{M} \rightarrow g$ locally uniformly in $\Omega \times R^{m} \times R^{k}$,

(c) $g_{M}(\mathbf{x}, \mathbf{u}, \mathbf{v}) \geqslant b(\mathbf{x})$, for some $b(\cdot) \in L^{1}(\Omega)$.

Let $\left\{\mathbf{u}_{\mathbf{M}}\right\}, \mathbf{u} \in L^{p}\left(\Omega ; R^{m}\right)$ and $\left\{\mathbf{v}_{\mathbf{M}}\right\}, \mathbf{v} \in L^{1}\left(\Omega ; R^{k}\right)$ be such that

$$
\mathbf{u}_{\mathbf{M}} \rightarrow \mathbf{u} \text { in } L^{p}\left(\Omega ; R^{m}\right) \text { and } \mathbf{v}_{\mathbf{M}} \rightarrow \mathbf{v} \text { in } L^{1}\left(\Omega ; R^{k}\right) .
$$

Then,

$$
\int_{\Omega} g(\mathbf{x}, \mathbf{u}, \mathbf{v}) d \mathbf{x} \leqslant \liminf _{M \rightarrow+\infty} \int_{\Omega} g_{M}\left(\mathbf{x}, \mathbf{u}_{\mathbf{M}}, \mathbf{v}_{\mathbf{M}}\right) d \mathbf{x} .
$$

Definition 2.4. [9] A function $f: R^{m n} \rightarrow \bar{R}$ is said to be polyconvex if there exists $g: R^{\tau(m, n)} \rightarrow \bar{R}$ convex, such that

$$
f(\mathbf{P})=g(\mathbf{T}(\mathbf{P})),
$$

where $\mathbf{T}: R^{m n} \rightarrow R^{\tau(m, n)}$ is given by $\mathbf{T}(\mathbf{P})=\left(\mathbf{P}, \operatorname{adj}_{2} \mathbf{P}, \cdots, \operatorname{adj}_{n \wedge m} \mathbf{P}\right)$.

In the preceding definition, $\operatorname{adj}_{s} \mathbf{P}$ stands for the matrix of all $s \times s$ minors of the matrix $\mathbf{P} \in R^{m n}, 2 \leqslant s \leqslant n \wedge m=\min \{n, m\}$, and

$$
\tau(m, n)=\sum_{s=1}^{n \wedge m} \sigma(s), \text { where } \sigma(s)=\frac{m ! n !}{(s !)^{2}(m-s) !(n-s) !} .
$$

Remark 2.1. Note that in the case $m=n=2$, the notion of (2.1) can be read as $f(\mathbf{P})=g(\mathbf{T}(\mathbf{P}))=g(\mathbf{P}, \operatorname{det} \mathbf{P})$.

We close this section with some results concerning the weak continuity of the $" \operatorname{adj}_{s} "$ functions $[9]$.

Theorem 2.2. Let $1<p<+\infty$, and $\mathbf{u}_{\mathbf{k}} \rightarrow \mathbf{u}$ in $W^{1, p}\left(\Omega ; R^{m}\right)$.

(i) Let $m, n \geqslant 2,2 \leqslant s \leqslant n \wedge m$ and $p \geqslant s$, then

$$
a d j_{s} D \mathbf{u}_{\mathbf{k}} \rightarrow a d j_{s} D \mathbf{u} \quad \text { in }\left(\mathcal{D}^{\prime}(\Omega)\right)^{\sigma(s)} .
$$


(ii) Let $m, n \geqslant 2,2 \leqslant s \leqslant n \wedge m$ and assume that

$$
a d j_{s-1} D \mathbf{u}_{\mathbf{k}} \rightarrow a d j_{s-1} D \mathbf{u} \text { in }\left(L^{r}(\Omega)\right)^{\sigma(s-1)},
$$

where $r>1$ with $\frac{1}{p}+\frac{1}{r} \leqslant 1$, then

$$
a d j_{s} D \mathbf{u}_{\mathbf{k}} \rightarrow a d j_{s} D \mathbf{u} \quad i n\left(\mathcal{D}^{\prime}(\Omega)\right)^{\sigma(s)} .
$$

Remark 2.2. Under the assumption of Theorem 2.2, it is easily seen that, if $m, n \geqslant 2, p>n \wedge m$, then, $\mathbf{u}_{\mathbf{k}} \rightarrow \mathbf{u}$ in $W^{1, p}\left(\Omega ; R^{m}\right) \operatorname{implies}_{\operatorname{adj}_{s}} D \mathbf{u}_{\mathbf{k}} \rightarrow$ $\operatorname{adj}_{s} D \mathbf{u}$ in $\left(L^{1}(\Omega)\right)^{\sigma(s)}$ for each $2 \leqslant s \leqslant n \wedge m$.

\section{The CONVERGENCE THEOREMS FOR THE TRUnCATION METHOD FOR POLYCONVEX INTEGRANDS}

Assume that the integrand $f: \Omega \times R^{m} \times R^{m n} \rightarrow \bar{R}$ satisfies the following hypotheses.

(H1) $f(\mathbf{x}, \mathbf{u}, \cdot)$ is polyconvex for all $(\mathbf{x}, \mathbf{u}) \in \Omega \times R^{m}$, i. e. there exists a function $g: \Omega \times R^{m} \times R^{\tau(m, n)} \rightarrow \bar{R}$ such that $f(\mathbf{x}, \mathbf{u}, \mathbf{P})=g(\mathbf{x}, \mathbf{u}, \mathbf{T}(\mathbf{P}))$ and $g(\mathbf{x}, \mathbf{u}, \cdot)$ is convex for all $(\mathbf{x}, \mathbf{u}) \in \Omega \times R^{m}$;

(H2) $g(\mathbf{x}, \mathbf{u}, \mathbf{v})$ is a Carathéodory function;

(H3) There exists an $a(\cdot) \in L^{1}(\Omega)$ such that $f(\mathbf{x}, \mathbf{u}, \mathbf{P})=g(\mathbf{x}, \mathbf{u}, \mathbf{T}(\mathbf{P})) \geqslant a(\mathbf{x})$ for all $(\mathbf{x}, \mathbf{u}, \mathbf{T}(\mathbf{P})) \in \Omega \times R^{m} \times R^{\tau(m, n)}$;

$(\mathrm{H} 4)$ Let $d_{K}(\mathbf{x})=\sup _{|\mathbf{u}| \leqslant K,|\mathbf{P}| \leqslant K}|f(\mathbf{x}, \mathbf{u}, \mathbf{P})|$, then $d_{K}(\cdot) \in L^{1}(\Omega)$.

By (H3), without loss of generality, we may assume that $f$ is non-negative. Let $\mathcal{T}^{h}$ be a regular triangulation [8] of $\Omega$ with $h$ being the mesh size and let $\bar{\Omega}_{h}=\bigcup_{K \in \mathcal{T}^{h}} \bar{K}$. Let $\mathcal{A}$ be a closed convex subset of $W^{1,1}\left(\Omega ; R^{m}\right)$, and let $\mathcal{A}_{h}$ be closed convex subsets of the finite element function spaces $\left\{\mathbf{u} \in C\left(\bar{\Omega} ; R^{m}\right)\right.$ : $\left.\mathbf{u}\right|_{K}$ is affine, $\left.\forall K \in \mathcal{T}^{h}\right\}$ satisfying the $W^{1, p}\left(\Omega ; R^{m}\right), 1 \leqslant p \leqslant+\infty$, approximating property, that is, for all $\mathbf{u} \in \mathcal{A} \cap W^{1, p}\left(\Omega ; R^{m}\right)$, there exist $\mathbf{u}_{h} \in \mathcal{A}_{h}$ such that

$$
\mathbf{u}_{h} \rightarrow \mathbf{u} \quad \text { in } W^{1, p}\left(\Omega ; R^{m}\right), \quad \text { as } h \rightarrow 0 ;
$$

and on the other hand, if the above convergence holds in weak topology for some $\mathbf{u} \in W^{1, p}\left(\Omega ; R^{m}\right)$ and a sequence $\mathbf{u}_{h} \in \mathcal{A}_{h}$, then we have $\mathbf{u} \in \mathcal{A}$. 
The application of the truncation method to computing the minimizer of $I(\cdot)$ in $\mathcal{A}^{p}$ leads to the finite problem of minimizing

$$
I_{M}\left(\mathbf{u}_{h}\right)=\int_{\Omega} f_{M}\left(\mathbf{x}, \mathbf{u}_{h}, D \mathbf{u}_{h}\right) d \mathbf{x}
$$

in $\mathcal{A}_{h}$, where the integrand $f$ is replaced by certain slower growth truncation functions $f_{M}$ on regions where the function $\mathbf{u}_{h}$, and especially its gradient $D \mathbf{u}_{h}$ is so large that the growth of the integrand may be out of control. In [2, 18], the convergence results of the truncation method for the case when $f$ is convex were obtained for some specially designed truncation functions. With similar techniques as used in [2], we establish below the convergence theorems of the truncation method for the case when $f$ is polyconvex.

Let $\left\{\mathcal{T}^{h_{M}}\right\}_{M=1}^{+\infty}$ be a given family of regular triangulations of $\Omega$ with $h_{M} \rightarrow 0$ as $M \rightarrow+\infty$.

Lemma 3.1. Let $1 \leqslant p<+\infty$. Let $\widetilde{\mathcal{T}}^{h_{M}}$ be subsets of $\mathcal{T}^{h_{M}}$ such that the sets $\widetilde{\Omega}_{h_{M}}=\bigcup_{K \in \widetilde{T}^{h_{M}}} \bar{K}$ satisfy

$$
\lim _{l \rightarrow \infty} \operatorname{meas}_{n}\left(\bigcup_{M=l}^{+\infty} \widetilde{\Omega}_{h_{M}}\right)=0
$$

Let the truncation function $g_{M}(\mathbf{x}, \mathbf{u}, \mathbf{T}(\mathbf{P}) ; p)=f_{M}(\mathbf{x}, \mathbf{u}, \mathbf{P} ; p)$ be defined by

$$
g_{M}(\mathbf{x}, \mathbf{u}, \mathbf{T}(\mathbf{P}) ; p)= \begin{cases}g(\mathbf{x}, \mathbf{u}, \mathbf{T}(\mathbf{P})), & \mathbf{x} \in \Omega \backslash \widetilde{\Omega}_{h_{M}}, \\ \min \left\{\alpha_{h_{M}}(\mathbf{x})\left(1+|\mathbf{P}|^{p}\right), g(\mathbf{x}, \mathbf{u}, \mathbf{T}(\mathbf{P}))\right\}, & \mathbf{x} \in \widetilde{\Omega}_{h_{M}},\end{cases}
$$

where $\alpha_{h_{M}}(\cdot) \in L^{\infty}(\Omega)$ and $\bar{\alpha}_{h_{M}} \geqslant \alpha_{h_{M}}(\mathbf{x}) \geqslant \alpha_{1}>0$ a.e. in $\Omega$. Then

(a) $g_{M}(\cdot, \cdot, \cdot ; p)$ are $L \otimes B$-measurable;

(b) $g_{M}(\mathbf{x}, \mathbf{u}, \mathbf{T}(\mathbf{P}) ; p) \geqslant b(\mathbf{x})$, for some $b(\cdot) \in L^{1}(\Omega)$;

(c) $g_{M} \rightarrow g$ locally uniformly in $\Omega \times R^{m} \times R^{\tau(m, n)}$.

Proof. Since both $g(\mathbf{x}, \mathbf{u}, \mathbf{T}(\mathbf{P}))$ and $\alpha_{h_{M}}(\mathbf{x})\left(1+|\mathbf{P}|^{p}\right)$ are Carathéodory functions and hence $L \otimes B$-measurable, it is not difficult to verify that $g_{M}(\cdot, \cdot, \cdot ; p)$ are $L \otimes B$ measurable.

If we define $b(\mathbf{x})=\min \left\{a(\mathbf{x}), \alpha_{1}\right\}$, then $b(\cdot) \in L^{1}(\Omega)$ and $g_{M}(\mathbf{x}, \mathbf{u}, \mathbf{T}(\mathbf{P}) ; p) \geq$ $b(\mathbf{x})$. 
To prove (c), let

$$
\Omega_{l}=\Omega \backslash\left(\bigcup_{M=l}^{+\infty} \widetilde{\Omega}_{h_{M}}\right) .
$$

It is obvious that $\Omega_{l}$ are measurable and $\Omega_{l} \subset \Omega$. By (3.2) and (3.4), we have

$$
\operatorname{meas}_{n}\left(\Omega \backslash \Omega_{l}\right)=\operatorname{meas}_{n}\left(\bigcup_{M=l}^{+\infty} \widetilde{\Omega}_{h_{M}}\right) \rightarrow 0 \text {, as } l \rightarrow+\infty \text {. }
$$

It follows from (3.3) and (3.4) that, for each $l$,

$$
g_{M}(\mathbf{x}, \mathbf{u}, \mathbf{T}(\mathbf{P}) ; p)=g(\mathbf{x}, \mathbf{u}, \mathbf{T}(\mathbf{P})), \forall \mathbf{x} \in \Omega_{l} \text { as long as } M>l .
$$

This completes the proof.

Corollary 3.1. Let $1 \leqslant p<+\infty$. Let $g_{M}$ be given by (3.3) with $\widetilde{\Omega}_{h_{M}}$ satisfying (3.2). Define

$$
I_{M}(\mathbf{u} ; p)=\int_{\Omega} f_{M}(\mathbf{x}, \mathbf{u}, D \mathbf{u} ; p) d \mathbf{x}=\int_{\Omega} g_{M}(\mathbf{x}, \mathbf{u}, \mathbf{T}(D \mathbf{u}) ; p) d \mathbf{x} .
$$

Let $\left\{\mathbf{u}_{M}\right\}, \mathbf{u} \in W^{1, p}\left(\Omega ; R^{m}\right)$ be such that

$$
\mathbf{u}_{M} \rightarrow \mathbf{u} \text { in } L^{p}\left(\Omega ; R^{m}\right), \quad \mathbf{T}\left(D \mathbf{u}_{M}\right) \rightarrow \mathbf{T}(D \mathbf{u}) \text { in } L^{1}\left(\Omega ; R^{m}\right), \text { as } M \rightarrow+\infty .
$$

Then,

$$
I(\mathbf{u}) \leq \liminf _{M \rightarrow+\infty} I_{M}\left(\mathbf{u}_{M} ; p\right)
$$

Proof. The conclusion follows directly from Theorem 2.1 and Lemma 3.1.

Definition 3.1. A function $\mathbf{u} \in W^{1, p}\left(\Omega ; R^{m}\right)(1 \leqslant p<+\infty)$ is said to be a partial regular function with singular set $E(\mathbf{u})$, if $D \mathbf{u} \in L^{\infty}\left(\Omega \backslash F ; R^{m n}\right)$ for any open set $F \supset E(\mathbf{u})$, and $D \mathbf{u} \notin L^{\infty}\left(G ; R^{m n}\right)$ for any open set $G$ such that $G \cap E(\mathbf{u}) \neq \emptyset$.

In the remainder of this paper, we denote by $E$ a set with zero $n$-dimensional Lebesgue measure and finite $(n-1)$-dimensional Hausdorff measure, especially we always assume that the singular set $E(\mathbf{u})$ in question satisfies meas ${ }_{n} E(\mathbf{u})=0$ and its $(n-1)$-dimensional Hausdorff measure is finite. 
Definition 3.2. A sequence of sets $\widetilde{\Omega}_{h_{M}}^{E}=\bigcup_{K \in \widetilde{\mathcal{T}}^{h_{M}(E)}} \bar{K}$, where $\widetilde{\mathcal{T}}^{h_{M}}(E) \subset \mathcal{T}^{h_{M}}$, is called an admissible finite element covering of a given set $E$ if there exist $0<C_{2}\left(h_{M}\right) \leqslant C_{1}\left(h_{M}\right)$ satisfying $\sum_{M=1}^{+\infty} C_{1}\left(h_{M}\right)<+\infty$ such that

(D1) $E \subset \widetilde{\Omega}_{h_{M}}^{E}$,

(D2) $\forall K \in \widetilde{\mathcal{T}}^{h_{M}}(E), \operatorname{dist}(E, K) \leqslant C_{1}\left(h_{M}\right)$,

(D3) $\forall K \notin \widetilde{\mathcal{T}}^{h_{M}}(E), \operatorname{dist}(E, K) \geqslant C_{2}\left(h_{M}\right)$,

where $\operatorname{dist}(E, K)$ is the Euclidean distance between the two sets.

Definition 3.3. Let $1 \leqslant p<+\infty$. A sequence of truncation functionals

$$
I_{M}^{E}(\mathbf{u} ; p)=\int_{\Omega} f_{M}^{E}(\mathbf{x}, \mathbf{u}, D \mathbf{u} ; p) d \mathbf{x}=\int_{\Omega} g_{M}^{E}(\mathbf{x}, \mathbf{u}, \mathbf{T}(D \mathbf{u}) ; p) d \mathbf{x}
$$

where the truncation functions $g_{M}^{E}(\mathbf{x}, \mathbf{u}, \mathbf{T}(D \mathbf{u}) ; p)=f_{M}^{E}(\mathbf{x}, \mathbf{u}, D \mathbf{u} ; p)$ are defined by

$$
g_{M}^{E}(\mathbf{x}, \mathbf{u}, \mathbf{T}(\mathbf{P}) ; p)= \begin{cases}g(\mathbf{x}, \mathbf{u}, \mathbf{T}(\mathbf{P})), & \mathbf{x} \in \Omega \backslash \widetilde{\Omega}_{h_{M}}^{E}, \\ \min \left\{\alpha_{h_{M}}(\mathbf{x})\left(1+|\mathbf{P}|^{p}\right), g(\mathbf{x}, \mathbf{u}, \mathbf{T}(\mathbf{P}))\right\}, & \mathbf{x} \in \widetilde{\Omega}_{h_{M}}^{E},\end{cases}
$$

with $\alpha_{h_{M}}(\cdot) \in L^{\infty}(\Omega)$ and $\bar{\alpha}_{h_{M}} \geqslant \alpha_{h_{M}}(\mathbf{x}) \geqslant \alpha_{1}>0$ a.e. in $\Omega$, is said to be consistent with the set $E$ if $\left\{\widetilde{\Omega}_{h_{M}}^{E}\right\}$ is an admissible finite element covering of $E$.

Lemma 3.2. Let $1 \leqslant p<+\infty$. Let $\widetilde{\mathbf{u}} \in W^{1, p}\left(\Omega ; R^{m}\right)$ be a partially regular function with singular set $E(\widetilde{\mathbf{u}}) \subset E$ and satisfy $f(\mathbf{x}, \widetilde{\mathbf{u}}, D \widetilde{\mathbf{u}}) \in L^{1}(\Omega)$. Let $I_{M}^{E}(\cdot ; p)$ be consistent with the set $E$ with $\left\{\widetilde{\Omega}_{h_{M}}^{E}\right\}$ being the corresponding admissible finite element covering of $E$. Let $\mathbf{u}_{h} \in \mathcal{A}_{h}$ satisfy

$$
\mathbf{u}_{h} \rightarrow \widetilde{\mathbf{u}} \text { in } W^{1, p}\left(\Omega ; R^{m}\right), \quad \text { as } h \rightarrow 0,
$$

and be uniformly bounded in $W^{1, \infty}\left(\Omega \backslash \widetilde{\Omega}_{h_{M}}^{E} ; R^{m}\right)$ for each $M$. Then, there exists a non-increasing function $M(\varepsilon)>0$, and a function $h(\varepsilon, M)>0$ with $h(\cdot, M)$ non-decreasing and $h(\varepsilon, \cdot)$ non-increasing, such that, for all $\varepsilon>0$,

$$
\left|I_{M}^{E}\left(\mathbf{u}_{h} ; p\right)-I(\widetilde{\mathbf{u}})\right|<\varepsilon, \quad \text { if } M>M(\varepsilon) \text { and } 0<h<h(\varepsilon, M) .
$$


Proof.

$$
\begin{aligned}
I_{M}^{E}\left(\mathbf{u}_{h} ; p\right)-I(\widetilde{\mathbf{u}})= & \int_{\Omega}\left[f_{M}^{E}(\mathbf{x}, \widetilde{\mathbf{u}}, D \widetilde{\mathbf{u}} ; p)-f(\mathbf{x}, \widetilde{\mathbf{u}}, D \widetilde{\mathbf{u}})\right] d \mathbf{x} \\
& +\int_{\Omega}\left[f_{M}^{E}\left(\mathbf{x}, \mathbf{u}_{h}, D \mathbf{u}_{h} ; p\right)-f_{M}^{E}(\mathbf{x}, \widetilde{\mathbf{u}}, D \widetilde{\mathbf{u}} ; p)\right] d \mathbf{x} \\
\triangleq & I_{1}(M)+I_{2}(h, M) .
\end{aligned}
$$

By (3.6) and $g(\mathbf{x}, \mathbf{u}, \mathbf{T}(D \mathbf{u}))=f(\mathbf{x}, \mathbf{u}, D \mathbf{u})$, we have

$$
\begin{aligned}
\left|I_{1}(M)\right| & =\left|\int_{\widetilde{\Omega}_{h_{M}}^{E}}\left[f_{M}^{E}(\mathbf{x}, \widetilde{\mathbf{u}}, D \widetilde{\mathbf{u}} ; p)-f(\mathbf{x}, \widetilde{\mathbf{u}}, D \widetilde{\mathbf{u}})\right] d \mathbf{x}\right| \\
& \leqslant 2 \int_{\widetilde{\Omega}_{h_{M}}^{E}}|f(\mathbf{x}, \widetilde{\mathbf{u}}, D \widetilde{\mathbf{u}})| d \mathbf{x} .
\end{aligned}
$$

It follows from $f(\mathbf{x}, \widetilde{\mathbf{u}}, D \widetilde{\mathbf{u}}) \in L^{1}(\Omega)$ that for any $\varepsilon>0$, there exists a $\delta_{1}(\varepsilon)>0$, such that

$$
\int_{\Omega^{\prime}}|f(\mathbf{x}, \widetilde{\mathbf{u}}, D \widetilde{\mathbf{u}})| d \mathbf{x}<\varepsilon, \quad \forall \Omega^{\prime} \subset \Omega \text { with } \operatorname{meas}_{n}\left(\Omega^{\prime}\right)<\delta_{1}(\varepsilon) .
$$

Since the $(n-1)$-dimensional Hausdorff measure of $E$ is finite and $\left\{\widetilde{\Omega}_{h_{M}}^{E}\right\}$ is an admissible finite element covering of $E$, we have $\lim _{M \rightarrow+\infty} \operatorname{meas}_{n}\left(\widetilde{\Omega}_{h_{M}}^{E}\right)=0$. Thus, by (3.9) and (3.10), there exists a non-increasing positive function $M(\cdot)$ such that

$$
\operatorname{meas}_{n}\left(\widetilde{\Omega}_{h_{M}}^{E}\right)<\delta_{1}\left(\frac{\varepsilon}{4}\right) \text { and }\left|I_{1}(M)\right|<\frac{\varepsilon}{2}, \quad \forall M>M(\varepsilon) .
$$

By (3.6), we have

$$
\begin{aligned}
I_{2}(h, M)= & \int_{\widetilde{\Omega}_{h_{M}}^{E}}\left[g_{M}^{E}\left(\mathbf{x}, \mathbf{u}_{h}, \mathbf{T}\left(D \mathbf{u}_{h}\right) ; p\right)-g_{M}^{E}(\mathbf{x}, \widetilde{\mathbf{u}}, \mathbf{T}(D \widetilde{\mathbf{u}}) ; p)\right] d \mathbf{x} \\
& +\int_{\Omega \backslash \widetilde{\Omega}_{h_{M}}^{E}}\left[g\left(\mathbf{x}, \mathbf{u}_{h}, \mathbf{T}\left(D \mathbf{u}_{h}\right)\right)-g(\mathbf{x}, \widetilde{\mathbf{u}}, \mathbf{T}(D \widetilde{\mathbf{u}}))\right] d \mathbf{x} \\
\triangleq & I_{21}(h, M)+I_{22}(h, M) .
\end{aligned}
$$

To estimate $I_{21}(h, M)$, we first notice that, as a consequence of $(3.7),\left|D \mathbf{u}_{h}\right|^{p}$ are equi-integrable on $\Omega$, and thus, for any $\varepsilon>0$ and given $\bar{\alpha}_{h_{M}} \geqslant \alpha_{1}>0$, there 
exists a $\delta_{2}(\varepsilon, M)>0$, such that, for any $\Omega^{\prime} \subset \Omega$, we have

$$
\int_{\Omega^{\prime}} \bar{\alpha}_{h_{M}}\left|D \mathbf{u}_{h}\right|^{p} d \mathbf{x}<\varepsilon, \quad \forall h>0 \quad \text { if } \operatorname{meas}_{n}\left(\Omega^{\prime}\right)<\delta_{2}(\varepsilon, M) .
$$

We claim that for any $\varepsilon>0, M>0$, there exists a $h_{1}(\varepsilon, M)>0$ with $h_{1}(\cdot, M)$ non-decreasing and $h_{1}(\varepsilon, \cdot)$ non-increasing, such that

$$
\left|I_{21}(h, M)\right|<\frac{\varepsilon}{4}, \quad \forall h \in\left(0, h_{1}(\varepsilon, M)\right) .
$$

Suppose otherwise. Then, there would be $\varepsilon_{0}>0, M_{0}>0$ and a decreasing sequence $\left\{h_{j}^{0}\right\}$ with $\lim _{j \rightarrow+\infty} h_{j}^{0}=0$ such that $\left|I_{21}\left(h_{j}^{0}, M_{0}\right)\right| \geqslant \frac{\varepsilon_{0}}{4}$ for all $j$. By (3.7), without loss of generality, we may assume

$$
\mathbf{u}_{h_{j}^{0}} \rightarrow \widetilde{\mathbf{u}} \text { and } D \mathbf{u}_{h_{j}^{0}} \rightarrow D \widetilde{\mathbf{u}} \text { a.e. in } \Omega
$$

and furthermore,

$$
\mathbf{T}\left(D \mathbf{u}_{h_{j}^{0}}\right) \rightarrow \mathbf{T}(D \widetilde{\mathbf{u}}) \text { a.e. in } \Omega .
$$

Thus, by (3.6) and (H2), we have

$$
\left[g_{M_{0}}^{E}\left(\mathbf{x}, \mathbf{u}_{h_{j}^{0}}, \mathbf{T}\left(D \mathbf{u}_{h_{j}^{0}}\right) ; p\right)-g_{M_{0}}^{E}(\mathbf{x}, \widetilde{\mathbf{u}}, \mathbf{T}(D \widetilde{\mathbf{u}}) ; p)\right] \rightarrow 0 \quad \text { a.e. } \mathbf{x} \in \Omega
$$

Let

$$
\begin{aligned}
G\left(\varepsilon_{0}, M_{0}, h_{j}^{0}\right)=\{\mathbf{x} \in \Omega: & \\
& \left.\left|g_{M_{0}}^{E}\left(\mathbf{x}, \mathbf{u}_{h_{j}^{0}}, \mathbf{T}\left(D \mathbf{u}_{h_{j}^{0}}\right) ; p\right)-g_{M_{0}}^{E}(\mathbf{x}, \widetilde{\mathbf{u}}, \mathbf{T}(D \widetilde{\mathbf{u}}) ; p)\right| \geqslant \frac{\varepsilon_{0}}{16 \operatorname{meas}_{n}(\Omega)}\right\} .
\end{aligned}
$$

By (3.14), there exists $J_{0}=J\left(\varepsilon_{0}, M_{0}\right)>0$, such that

$$
\operatorname{meas}_{n}\left(G\left(\varepsilon_{0}, M_{0}, h_{j}^{0}\right)\right)<\min \left\{\frac{\varepsilon_{0}}{16 \bar{\alpha}_{h_{M_{0}}}}, \delta_{1}\left(\frac{\varepsilon_{0}}{16}\right), \delta_{2}\left(\frac{\varepsilon_{0}}{16}, M_{0}\right)\right\}, \quad \forall j>J_{0}
$$


As a consequence of (3.6), (3.10), (3.12) and (3.15), we have

$$
\begin{aligned}
& \left|I_{21}\left(h_{j}^{0}, M_{0}\right)\right| \leqslant \int_{\widetilde{\Omega}_{h_{M_{0}}}^{E} \cap G\left(\varepsilon_{0}, M_{0}, h_{j}^{0}\right)}\left[\bar{\alpha}_{h_{M_{0}}}\left(1+\left|D \mathbf{u}_{h_{j}^{0}}\right|^{p}\right)+|f(\mathbf{x}, \widetilde{\mathbf{u}}, D \widetilde{\mathbf{u}})|\right] d \mathbf{x} \\
& +\int_{\widetilde{\Omega}_{h_{M_{0}}}^{E} \cap\left(\Omega \backslash G\left(\varepsilon_{0}, M_{0}, h_{j}^{0}\right)\right)}\left|g_{M_{0}}^{E}\left(\mathbf{x}, \mathbf{u}_{h_{j}^{0}}, \mathbf{T}\left(D \mathbf{u}_{h_{j}^{0}}\right) ; p\right)-g_{M_{0}}^{E}(\mathbf{x}, \widetilde{\mathbf{u}}, \mathbf{T}(D \widetilde{\mathbf{u}}) ; p)\right| d \mathbf{x} \\
& \leqslant \frac{\varepsilon_{0}}{16 \bar{\alpha}_{h_{M_{0}}}} \bar{\alpha}_{h_{M_{0}}}+\frac{\varepsilon_{0}}{8}+\frac{\varepsilon_{0}}{16 \operatorname{meas}_{n}(\Omega)} \operatorname{meas}_{n}\left(\Omega \backslash G\left(\varepsilon_{0}, M_{0}, h_{j}^{0}\right)\right) \\
& <\frac{\varepsilon_{0}}{4}, \quad \forall j>J_{0} .
\end{aligned}
$$

This is a contradiction.

We also claim that for any $\varepsilon>0, M>0$, there exists $h_{2}(\varepsilon, M)>0$ with $h_{2}(\cdot, M)$ non-decreasing and $h_{2}(\varepsilon, \cdot)$ non-increasing, such that

$$
\left|I_{22}(h, M)\right|<\frac{\varepsilon}{4}, \quad \forall h \in\left(0, h_{2}(\varepsilon, M)\right) .
$$

Suppose otherwise. Then, there would be $\varepsilon_{1}>0, M_{1}>0$, and a decreasing sequence $\left\{h_{j}^{1}\right\}$ with $\lim _{j \rightarrow+\infty} h_{j}^{1}=0$ such that $\left|I_{22}\left(h_{j}^{1}, M_{1}\right)\right| \geqslant \frac{\varepsilon_{1}}{4}$ for all $j$. By (3.7), without loss of generality, we may assume that

$$
\mathbf{u}_{h_{j}^{1}} \rightarrow \widetilde{\mathbf{u}} \text { and } D \mathbf{u}_{h_{j}^{1}} \rightarrow D \widetilde{\mathbf{u}} \text { a.e. in } \Omega
$$

and

$$
\mathbf{T}\left(D \mathbf{u}_{h_{j}^{1}}\right) \rightarrow \mathbf{T}(D \widetilde{\mathbf{u}}) \text { a.e. in } \Omega
$$

and thus, by (H2), we have

$$
\left[g\left(\mathbf{x}, \mathbf{u}_{h_{j}^{1}}, \mathbf{T}\left(D \mathbf{u}_{h_{j}^{1}}\right)\right)-g(\mathbf{x}, \widetilde{\mathbf{u}}, \mathbf{T}(D \widetilde{\mathbf{u}}))\right] \rightarrow 0 \text { a.e. } \mathbf{x} \in \Omega .
$$

By (H4), and noticing that by assumption there exists a $C\left(M_{1}\right)>0$ such that

$$
\left|\mathbf{u}_{h}(\mathbf{x})\right| \leqslant C\left(M_{1}\right) \text { and }\left|D \mathbf{u}_{h}(\mathbf{x})\right| \leqslant C\left(M_{1}\right) \text { a.e. } \mathbf{x} \in \Omega \backslash \widetilde{\Omega}_{h_{M_{1}}}^{E}, \forall h,
$$

we have

$$
\begin{array}{r}
\left|g\left(\mathbf{x}, \mathbf{u}_{h_{j}^{1}}, \mathbf{T}\left(D \mathbf{u}_{h_{j}^{1}}\right)\right)-g(\mathbf{x}, \widetilde{\mathbf{u}}, \mathbf{T}(D \widetilde{\mathbf{u}}))\right|=\left|f\left(\mathbf{x}, \mathbf{u}_{h_{j}^{1}}, D \mathbf{u}_{h_{j}^{1}}\right)-f(\mathbf{x}, \widetilde{\mathbf{u}}, D \widetilde{\mathbf{u}})\right| \\
\leqslant d_{C\left(M_{1}\right)}(\mathbf{x})+|f(\mathbf{x}, \widetilde{\mathbf{u}}, D \widetilde{\mathbf{u}})| \in L^{1}\left(\Omega \backslash \widetilde{\Omega}_{h_{M_{1}}}^{E}\right) .
\end{array}
$$


It follows from (3.18), (3.19) and the dominated convergence theorem [14] that

$$
\lim _{j \rightarrow+\infty}\left|I_{22}\left(h_{j}^{1}, M_{1}\right)\right|=0 .
$$

This is a contradiction.

Now, (3.8) follows as a consequence of (3.11), (3.13) and (3.17) by setting $h(\varepsilon, M)=\min \left\{h_{1}(\varepsilon, M), h_{2}(\varepsilon, M), h_{M}\right\}$. This completes the proof.

Theorem 3.1. Let $1 \leqslant p<+\infty$. Let

$$
\mathcal{A}_{E}^{p}=\left\{\mathbf{u} \in \mathcal{A}^{p}: \mathbf{u} \text { is a partially regular function with singular set } E(\mathbf{u}) \subset E\right\} \text {. }
$$

Let $I_{M}^{E}(\cdot ; p)$ be consistent with the singular set $E$ with $\left\{\widetilde{\Omega}_{h_{M}}^{E}\right\}$ being the corresponding admissible finite element covering of $E$. Then, there exists a non-increasing function $M(\varepsilon)>0$, and a function $h(\varepsilon, M)>0$ with $h(\cdot, M)$ non-decreasing and $h(\varepsilon, \cdot)$ non-increasing, such that, for all $\varepsilon>0$,

$$
\inf _{\mathbf{u}_{h} \in \mathcal{A}_{h}} I_{M}^{E}\left(\mathbf{u}_{h} ; p\right)<\inf _{\mathbf{u} \in \mathcal{A}_{E}^{p}} I(\mathbf{u})+2 \varepsilon, \quad \text { if } M>M(\varepsilon) \text { and } 0<h<h(\varepsilon, M) .
$$

Proof. Without loss of generality, we assume that, for any $\varepsilon>0$, there exists a $\widetilde{\mathbf{u}}_{\varepsilon} \in \mathcal{A}_{E}^{p}$ such that

$$
I\left(\widetilde{\mathbf{u}}_{\varepsilon}\right)<\inf _{\mathbf{u} \in \mathcal{A}_{E}^{p}} I(\mathbf{u})+\varepsilon<+\infty .
$$

Extending $\widetilde{\mathbf{u}}_{\varepsilon}$ to $W_{0}^{1, p}\left(R^{n} ; R^{m}\right)$ by the extension theorem for Sobolev spaces [1], recalling that $\widetilde{\mathbf{u}}_{\varepsilon} \in W^{1, \infty}\left(\Omega \backslash F ; R^{m}\right)$ for any open set $E \subset F \subset \Omega$, we may assume that $\widetilde{\mathbf{u}}_{\varepsilon} \in W^{1, \infty}\left(R^{n} \backslash F ; R^{m}\right)$ for any open set $E \subset F \subset R^{n}$. Thus, by the denseness of smooth functions in $W_{0}^{1, p}\left(R^{n} ; R^{m}\right)$ [1] and the standard finite approximation theories [8], there exist $\mathbf{u}_{h}^{\varepsilon} \in \mathcal{A}_{h}$ such that $\mathbf{u}_{h}^{\varepsilon}$ are uniformly bounded in $W^{1, \infty}\left(\Omega \backslash \widetilde{\Omega}_{h_{M}}^{E} ; R^{m}\right)$ for each $M$ and

$$
\mathbf{u}_{h}^{\varepsilon} \rightarrow \widetilde{\mathbf{u}}_{\varepsilon} \text { in } W^{1, p}\left(\Omega ; R^{m}\right), \quad \text { as } h \rightarrow 0 .
$$

Hence, from Lemma 3.2 and (3.22), the conclusion of the theorem follows.

Our main results are the following two theorems, which, briefly speaking, conclude that the truncation method converges in the case when the absolute minimizer exists (Theorem 3.2), and it leads to a minimizing sequence if the infimum is unattainable (Theorem 3.3). 
Theorem 3.2. Suppose that $\hat{\mathbf{u}} \in W^{1, q}\left(\Omega ; R^{m}\right)(1 \leqslant q<+\infty)$ is a minimizer of $I(\cdot)$ in $\mathcal{A}^{p}(q \geqslant p>n \wedge m)$ and $\hat{\mathbf{u}} \in \mathcal{A}_{E}^{p}$ (see (3.20)). Let $\left\{\varepsilon_{j}\right\}$ be a decreasing sequence with $\lim _{j \rightarrow \infty} \varepsilon_{j}=0$. Let $I_{M}^{E}(\cdot ; p)$ be consistent with the set $E$, with $\left\{\widetilde{\Omega}_{h_{M}}^{E}\right\}$ being the corresponding admissible finite element covering of $E$. Then

(1) There exist a non-increasing function $M(\varepsilon)>0$ and a function $h(\varepsilon, M)>$ 0 with $h(\cdot, M)$ non-decreasing and $h(\varepsilon, \cdot)$ non-increasing such that

$$
\inf _{\mathbf{u} \in \mathcal{A}_{h}} I_{M}^{E}(\mathbf{u} ; p)<I(\hat{\mathbf{u}})+\varepsilon_{j}, \forall M \geqslant M\left(\varepsilon_{j}\right) \text { and } \forall h \in\left(0, h\left(\varepsilon_{j}, M\right)\right)
$$

and, for all $M \geqslant M\left(\varepsilon_{j}\right)$ and $0<h \leqslant h\left(\varepsilon_{j}, M\right)$, there exist $\mathbf{u}_{h}^{\varepsilon_{j}} \in \mathcal{A}_{h}$ such that $\mathbf{u}_{h}^{\varepsilon_{j}}$ are uniformly bounded in $W^{1, \infty}\left(\Omega \backslash \widetilde{\Omega}_{h_{M}}^{E} ; R^{m}\right)$ for each $M$ and

$$
I_{M}^{E}\left(\mathbf{u}_{h}^{\varepsilon_{j}} ; p\right)<I(\hat{\mathbf{u}})+2 \varepsilon_{j}, \forall M \geqslant M\left(\varepsilon_{j}\right) \text { and } \forall h \in\left(0, h\left(\varepsilon_{j}, M\right)\right) .
$$

(2) Let $M_{j} \geqslant M\left(\varepsilon_{j}\right), 0<h^{j} \leqslant h\left(\varepsilon_{j}, M_{j}\right)$. Let $\overline{\mathbf{u}}_{j} \in \mathcal{A}_{h^{j}}$ be minimizers of $I_{M_{j}}^{E}(\cdot ; p)$ in $\mathcal{A}_{h^{j}}$. Suppose that the sequence $\left\{\overline{\mathbf{u}}_{j}\right\}_{j=1}^{+\infty}$ is sequentially weakly precompact in $W^{1, r}\left(\Omega ; R^{m}\right)$ for some $p \leqslant r \leqslant q$. Then, there exists a function $\overline{\mathbf{u}} \in \mathcal{A}^{r}$, and a subsequence of $\left\{\overline{\mathbf{u}}_{j}\right\}_{j=1}^{+\infty}$, again denoted by $\left\{\overline{\mathbf{u}}_{j}\right\}_{j=1}^{+\infty}$, such that

$$
\overline{\mathbf{u}}_{j} \rightarrow \overline{\mathbf{u}} \text { in } W^{1, r}\left(\Omega ; R^{m}\right)
$$

and

$$
I(\overline{\mathbf{u}})=\inf _{\mathbf{u} \in \mathcal{A}^{p}} I(\mathbf{u})=\lim _{j \rightarrow+\infty} I_{M_{j}}^{E}\left(\overline{\mathbf{u}}_{j} ; p\right)
$$

Proof. The conclusion (1) of the theorem follows from a similar argument as in the proof of Theorem 3.1 .

(3.25) is a consequence of the sequentially weak precompactness of the sequence $\left\{\overline{\mathbf{u}}_{j}\right\}_{j=1}^{+\infty}$, and it follows from the approximating property of $\mathcal{A}_{h}$ that $\overline{\mathbf{u}} \in \mathcal{A}^{r}$. Because of $r \geqslant p>n \wedge m$, we can use Theorem 2.2 to obtain

$$
\mathbf{T}\left(D \overline{\mathbf{u}}_{j}\right) \rightarrow \mathbf{T}(D \overline{\mathbf{u}}), \quad \text { in } \quad L^{1}\left(\Omega ; R^{\tau(m, n)}\right) .
$$

Then, by Corollary 3.1, we have

$$
I(\overline{\mathbf{u}}) \leqslant \liminf _{j \rightarrow+\infty} I_{M_{j}}^{E}\left(\overline{\mathbf{u}}_{j} ; p\right) .
$$


On the other hand, by (3.23), we have

$$
I_{M_{j}}^{E}\left(\overline{\mathbf{u}}_{j} ; p\right)=\inf _{\mathbf{u} \in \mathcal{A}_{h j}} I_{M_{j}}^{E}(\mathbf{u} ; p)<I(\hat{\mathbf{u}})+\varepsilon_{j},
$$

and thus,

$$
\limsup _{j \rightarrow+\infty} I_{M_{j}}^{E}\left(\overline{\mathbf{u}}_{j} ; p\right) \leqslant I(\hat{\mathbf{u}})=\inf _{\mathbf{u} \in \mathcal{A}^{p}} I(\mathbf{u}) .
$$

This, and (3.27) lead to (3.26).

Remark 3.1. When $r>1$, the boundedness of $\left\{\overline{\mathbf{u}}_{j}\right\}_{j=1}^{+\infty}$ in $W^{1, r}\left(\Omega ; R^{m}\right)$ implies that the sequence is sequentially weak precompact in $W^{1, r}\left(\Omega ; R^{m}\right)$.

Theorem 3.3. Let $(n \wedge m)<p<+\infty$. Let $\left\{L_{i}\right\}_{i=1}^{+\infty}$ be an increasing sequence satisfying $\lim _{i \rightarrow+\infty} L_{i}=+\infty$. Define

$$
\begin{aligned}
& \mathcal{A}^{p}\left(L_{i}\right)=\left\{\mathbf{u} \in \mathcal{A}^{p}:|\mathbf{u}|_{1, p}^{p} \leqslant L_{i}\right\}, \\
& \mathcal{A}_{h}^{p}\left(L_{i}\right)=\left\{\mathbf{u} \in \mathcal{A}_{h}:|\mathbf{u}|_{1, p}^{p} \leqslant L_{i}\right\} .
\end{aligned}
$$

For each $i \in \mathcal{N}$, suppose that $\hat{\mathbf{u}}^{i} \in \mathcal{A}_{E}^{p}$ (see (3.20)) is a minimizer of $I(\cdot)$ in $\mathcal{A}^{p}\left(L_{i}\right)$. Let $\left\{\varepsilon_{j}\right\}$ be a decreasing sequence with $\lim _{j \rightarrow \infty} \varepsilon_{j}=0$. Then, for each $i \in \mathcal{N}$,

(1) There exist a non-increasing function $M(\varepsilon)>0$ and a function $h(\varepsilon, M)>$ 0 with $h(\cdot, M)$ non-decreasing and $h(\varepsilon, \cdot)$ non-increasing such that

$$
\inf _{\mathbf{u} \in \mathcal{A}_{h}^{p}\left(L_{i}\right)} I_{M}^{E}(\mathbf{u} ; p)<I\left(\hat{\mathbf{u}}^{i}\right)+\varepsilon_{j}, \quad \text { if } M \geqslant M\left(\varepsilon_{j}\right) \text { and } 0<h \leqslant h\left(\varepsilon_{j}, M\right) .
$$

(2) Let $M_{j} \geqslant M\left(\varepsilon_{j}\right), 0<h^{j} \leqslant h\left(\varepsilon_{j}, M_{j}\right)$. Let $\overline{\mathbf{u}}_{j}^{i} \in \mathcal{A}_{h^{j}}^{p}\left(L_{i}\right)$ be minimizers of $I_{M_{j}}^{E}(\cdot ; p)$ in $\mathcal{A}_{h^{j}}^{p}\left(L_{i}\right)$. Then there exist a function $\overline{\mathbf{u}}^{i} \in \mathcal{A}^{p}\left(L_{i}\right)$ and a subsequence of $\left\{\overline{\mathbf{u}}_{j}^{i}\right\}_{j=1}^{+\infty}$, again denoted by $\left\{\overline{\mathbf{u}}_{j}^{i}\right\}_{j=1}^{+\infty}$, such that

$$
\overline{\mathbf{u}}_{j}^{i} \rightarrow \overline{\mathbf{u}}^{i} \text { in } W^{1, p}\left(\Omega ; R^{m}\right), \quad \text { as } j \rightarrow+\infty,
$$

and

$$
I\left(\overline{\mathbf{u}}^{i}\right)=\inf _{\mathbf{u} \in \mathcal{A}^{p}\left(L_{i}\right)} I(\mathbf{u})=\lim _{j \rightarrow+\infty} I_{M_{j}}^{E}\left(\overline{\mathbf{u}}_{j}^{i} ; p\right) .
$$

(3) There exists a non-decreasing function $j(i)$ satisfying $\lim _{i \rightarrow+\infty} j(i)=+\infty$ such that

$$
\inf _{\mathbf{u} \in \mathcal{A}^{p}} I(\mathbf{u})=\lim _{i \rightarrow+\infty} I\left(\overline{\mathbf{u}}^{i}\right)=\lim _{i \rightarrow+\infty} I_{M_{j(i)}}^{E}\left(\overline{\mathbf{u}}_{j(i)}^{i} ; p\right)
$$


Proof. For each $i \in \mathcal{N}$, the conclusion (1) and (2) of the theorem follow from a similar argument as in the proof of Theorem 3.2. The conclusion (3) of the theorem follows from (3.29) and (3.32).

Remark 3.2. The singular set $E(\mathbf{u})$ for an absolute minimizer is usually not known in advance when the Lavrentiev phenomenon is involved, and thus it needs to be decided in the process of computation by taking some initial guesses and comparing the numerical results thus produced. An element is finally taken into the set $\widetilde{\Omega}_{h_{M}}^{E}$, if the inclusion leads to a substantial increase of the gradient of the numerical solution on the element in the minimizing process, otherwise it is removed from the initial guess. How to find efficiently a good initial guess is an open problem. Fortunately, in applications, $E(\mathbf{u})$ is usually contained in a set $E$ where the standard finite element solutions have large derivatives.

Remark 3.3. The approximating property of the finite element function spaces $\mathcal{A}_{h}$ to $\mathcal{A}$ is easily satisfied in applications. Especially, it covers problems with Dirichlet boundary conditions and the examples in nonlinear elasticity given by Foss et. al. [13], which are used in our numerical experiments shown in the next section.

\section{NumERICAL RESUltS ON EXAMPLES IN NONLINEAR ELASTICITY}

For the convenience of the reader, we first review some theoretical results of Foss et. al. [13] on some problems of nonlinear elasticity exhibiting the Lavrentiev phenomenon.

4.1. Examples in nonlinear elasticity [13]. Consider the stored energy density $W_{0}: \operatorname{Lin}\left(R^{2} ; R^{2}\right) \rightarrow R$ defined by

$$
W_{0}(\mathbf{P})=\left[\|\mathbf{P}\|^{2}-2 \operatorname{det} \mathbf{P}\right]^{4},
$$

where $\|\mathbf{P}\|^{2}=\operatorname{tr}\left(\mathbf{P P}^{T}\right)$ and $\operatorname{Lin}\left(R^{2} ; R^{2}\right)$ denotes the set of linear operators from $R^{2}$ to $R^{2}$, and consider another stored energy density $W_{\varepsilon, \kappa}: \operatorname{Lin}^{+}\left(R^{2} ; R^{2}\right) \rightarrow R$, which is a perturbation of $W_{0}$, defined by

$$
W_{\varepsilon, \kappa}(\mathbf{P})=W_{0}(\mathbf{P})+\varepsilon\left[\frac{\kappa}{\operatorname{det} \mathbf{P}}+3^{\frac{2-\kappa}{2}}(1+\|\mathbf{P}\|)^{\frac{\kappa}{2}}\right],
$$

where $\operatorname{Lin}^{+}\left(R^{2} ; R^{2}\right)$ is a subset of $\operatorname{Lin}\left(R^{2} ; R^{2}\right)$ with elements of positive determinant. Foss et. al. [13] showed the following results: 
Theorem 4.1. Let $W_{0}$ given by (4.1), then

$\left(a_{0}\right) W_{0} \in C^{\infty}\left(\operatorname{Lin}\left(R^{2} ; R^{2}\right) ; R\right)$ is materially homogeneous, frame-indifferent and isotropic;

$\left(b_{0}\right) W_{0}$ is convex over $\operatorname{Lin}\left(R^{2} ; R^{2}\right)$ and $W_{0}(\mathbf{P}) \geqslant 0$ at each $\mathbf{P} \in \operatorname{Lin}\left(R^{2} ; R^{2}\right)$.

Let $W_{\varepsilon, \kappa}$ be given by (4.2), then, for all $\varepsilon>0$ and $\kappa \geqslant 2$,

$\left(a_{\varepsilon, \kappa}\right) W_{\varepsilon, \kappa} \in C^{\infty}\left(\operatorname{Lin}^{+}\left(R^{2} ; R^{2}\right) ; R\right)$ is materially homogeneous, frame-indifferent and isotropic;

$\left(b_{\varepsilon, \kappa}\right) W_{\varepsilon, \kappa}$ is polyconvex and $W_{\varepsilon, \kappa}(\mathbf{P}) \geqslant \varepsilon\|\mathbf{P}\|^{\kappa}, \forall \mathbf{P} \in \operatorname{Lin}^{+}\left(R^{2} ; R^{2}\right)$;

$\left(c_{\varepsilon, \kappa}\right) W_{\varepsilon, \kappa}(\mathbf{P}) \rightarrow+\infty$ as $\operatorname{det} \mathbf{P} \rightarrow 0^{+}$.

Consider the reference and deformed configuration of the form

$$
\Omega_{\alpha}=\left\{\mathbf{x} \in R^{2}: r(\mathbf{x})<1 \text { and } \vartheta(\mathbf{x}) \in(0, \alpha)\right\}
$$

for $\alpha \in(0,2 \pi)$, where $r(\mathbf{x})$ and $\vartheta(\mathbf{x})$ are the magnitude and the polar angle of the vector $\mathrm{x} \in R^{2}$ respectively. Partition the boundary of $\Omega_{\alpha}$ as follows:

$$
\begin{aligned}
& \Gamma_{1, \alpha}=\left\{\mathbf{x} \in \partial \Omega_{\alpha}: r(\mathbf{x}) \leqslant 1 \text { and } \vartheta(\mathbf{x})=\alpha\right\} ; \\
& \Gamma_{2, \alpha}=\left\{\mathbf{x} \in \partial \Omega_{\alpha}: r(\mathbf{x}) \leqslant 1 \text { and } \vartheta(\mathbf{x})=0\right\} ; \\
& \Gamma_{3, \alpha}=\left\{\mathbf{x} \in \partial \Omega_{\alpha}: r(\mathbf{x})=1\right\} .
\end{aligned}
$$

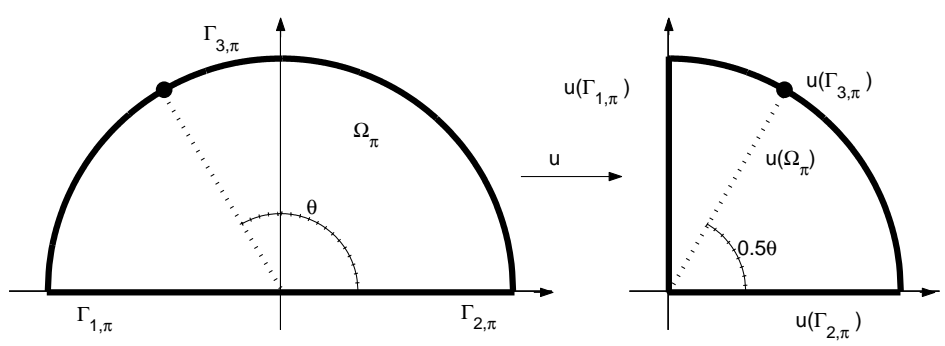

Figure 1. The boundary conditions $\left(B C_{\pi, \frac{\pi}{2}}\right)$.

A mapping $\mathbf{u} \in C\left(\bar{\Omega}_{\pi} ; R^{2}\right)$ is said to satisfy the boundary conditions $\left(B C_{\pi, \frac{\pi}{2}}\right)$ if (see Figure 1)

$$
\begin{aligned}
& \left(1_{\pi, \frac{\pi}{2}}\right) \mathbf{u}\left(\Gamma_{1, \pi}\right)=\Gamma_{1, \frac{\pi}{2}} \\
& \left(2_{\pi, \frac{\pi}{2}}\right) \mathbf{u}\left(\Gamma_{2, \pi}\right)=\Gamma_{2, \frac{\pi}{2}} ; \\
& \left(3_{\pi, \frac{\pi}{2}}\right) r(\mathbf{u}(\mathbf{x}))=1 \text { and } \vartheta(\mathbf{u}(\mathbf{x}))=\frac{1}{2} \vartheta(\mathbf{x}), \forall \mathbf{x} \in \Gamma_{3, \pi} .
\end{aligned}
$$


Consider the admissible deformations of the form

$$
\begin{aligned}
\mathcal{A}_{\pi, \frac{\pi}{2}}^{1}=\left\{\mathbf{u} \in W^{1,1}\left(\Omega_{\pi} ; R^{2}\right)\right. & \cap C\left(\bar{\Omega}_{\pi} ; R^{2}\right), \\
& \left.\mathbf{u} \text { satisfies }\left(B C_{\pi, \frac{\pi}{2}}\right) \text { and } \operatorname{det} D \mathbf{u}(\mathbf{x})>0 \quad \mathbf{x} \in \Omega_{\pi}\right\} .
\end{aligned}
$$

It is easily seen that for $1 \leqslant p \leqslant+\infty$

$$
\mathcal{A}_{\pi, \frac{\pi}{2}}^{p}=\mathcal{A}_{\pi, \frac{\pi}{2}}^{1} \cap W^{1, p}\left(\Omega_{\pi} ; R^{2}\right) .
$$

Define the functional $I_{0}(\mathbf{u}): W^{1,1}\left(\Omega_{\pi} ; R^{2}\right) \rightarrow \bar{R}$ by

$$
I_{0}(\mathbf{u})=\int_{\Omega_{\pi}} W_{0}(D \mathbf{u}(\mathbf{x})) d \mathbf{x}
$$

For $\varepsilon>0$ and $\kappa>2$, define $I_{\varepsilon, \kappa}(\mathbf{u}): W^{1,1}\left(\Omega_{\pi} ; R^{2}\right) \rightarrow \bar{R}$ by

$$
I_{\varepsilon, \kappa}(\mathbf{u})=\int_{\Omega_{\pi}} W_{\varepsilon, \kappa}(D \mathbf{u}(\mathbf{x})) d \mathbf{x}
$$

The following theorem shows that $I_{0}$ and $I_{\varepsilon, \kappa}$ exhibit the Lavrentiev phenomenon (see [13] for more general results).

Theorem 4.2. If $p_{1}, p_{2} \in[1,+\infty]$ satisfy $p_{1}<4<p_{2}$, then

$$
\inf _{u \in \mathcal{A}_{\pi, \frac{\pi}{2}}^{p_{2}}} I_{0}(\mathbf{u}) \geq I_{0}\left(\mathbf{u}_{p m}(\mathbf{x})\right)=\left(\frac{2}{7}\right)^{7} \pi>0=I_{0}\left(\mathbf{u}_{a m}(\mathbf{x})\right)=\inf _{\mathbf{u} \in \mathcal{A}_{\pi, \frac{\pi}{2}}^{p_{1}}} I_{0}(\mathbf{u}),
$$

where $\mathbf{u}_{a m} \in \mathcal{A}_{\pi, \frac{\pi}{2}}^{q_{1}}$ with $q_{1} \in[1,4)$ and $\mathbf{u}_{p m} \in \mathcal{A}_{\pi, \frac{\pi}{2}}^{q_{2}}$ with $q_{2} \in\left[4, \frac{28}{3}\right)$ are given respectively by

$$
\begin{aligned}
& \mathbf{u}_{a m}(\mathbf{x})=r(\mathbf{x})^{\frac{1}{2}}\left(\begin{array}{c}
\cos \left(\frac{1}{2} \vartheta(\mathbf{x})\right) \\
\sin \left(\frac{1}{2} \vartheta(\mathbf{x})\right)
\end{array}\right), \\
& \mathbf{u}_{p m}(\mathbf{x})=r(\mathbf{x})^{\frac{11}{14}}\left(\begin{array}{c}
\cos \left(\frac{1}{2} \vartheta(\mathbf{x})\right) \\
\sin \left(\frac{1}{2} \vartheta(\mathbf{x})\right)
\end{array}\right) .
\end{aligned}
$$

(2) If $2 \leqslant \kappa<4$ and $0<\varepsilon<\varepsilon_{\pi, \frac{\pi}{2}, \kappa}=\left(\frac{2}{7}\right)^{7}\left(F_{k}\left(\mathbf{u}_{a m}\right)\right)^{-1}$, we have

$$
\inf _{\mathbf{u} \in \mathcal{A}_{\pi, \frac{\pi}{2}}^{p_{2}}} I_{\varepsilon, \kappa}(\mathbf{u})>\inf _{\mathbf{u} \in \mathcal{A}_{\pi, \frac{\pi}{2}}^{p_{1}}} I_{\varepsilon, \kappa}(\mathbf{u})
$$




$$
\text { where } F_{k}\left(\mathbf{u}_{a m}\right)=\int_{\Omega_{\pi}}\left[\frac{\kappa}{\operatorname{det} D \mathbf{u}_{a m}}+3^{\frac{2-\kappa}{2}}\left(1+\left\|D \mathbf{u}_{a m}\right\|\right)^{\frac{\kappa}{2}}\right] d \mathbf{x} \text {. }
$$

4.2. Some tips for the numerical experiments. According to the convergence theory developed in section 3 , in a numerical experiment, we need to determine $h_{M}, \alpha_{h_{M}}$ and $\widetilde{\Omega}_{h_{M}}^{E}$, where $M$ is only a index which we do not really need to take too much care. We summarize our experience as follows, which may be helpful to the readers:

(1): The size of $h_{M}$ should be taken to balance the precision and cost of the numerical computation. Since that, in general, the structure of the singular set $E$ is not known, we need to compare the numerical results obtained with different $h_{M}$ to see if the numerical singular sets converge.

(2): In our numerical experiments, the initial guess of $\widetilde{\Omega}_{h_{M}}^{E}$ is determined by including into the set the elements on which the numerical solution obtained by the standard finite element method has large derivatives. Then, after applying the truncation method, the set $\widetilde{\Omega}_{h_{M}}^{E}$ is modified by removing the elements where the truncation solution derivatives drop and adding in the elements where the truncation solution derivatives increase significantly. Further modification of the set $\widetilde{\Omega}_{h_{M}}^{E}$ can be made by comparing the truncation solution derivatives on each element with the derivatives of the truncation solutions obtained on a refined mesh, removing or adding in an element according to the tendency of the growth of the derivatives. The modification process usually needs to be repeated a few times before a stable set $\widetilde{\Omega}_{h_{M}}^{E}$ is finally obtained. Even though there is no theory to guarantee that this works in general, numerical experiments showed that it worked well on known examples exhibiting the Lavrentiev phenomenon. (3): The choice of $\alpha_{h_{M}}$ is crucial to the accuracy and efficiency of the computation. It is obvious that the truncation method does not work, if $\alpha_{h_{M}}$ is too large. On the other hand, if $\alpha_{h_{M}}$ is too small, then the derivatives of the truncation solution may have a very big jump across the boundary of the set $\widetilde{\Omega}_{h_{M}}^{E}$. The general principle is to lower the minimum energy of $I_{M}^{E}$ while keeping certain smoothness of the numerical solution $\mathbf{u}_{h}$. The determination of $\alpha_{h_{M}}$ can usually be combined with a process of numerically estimating the leading order of the singularity of the minimizer $\mathbf{u}_{h}$, 
which involves constructing in a superset of $\widetilde{\Omega}_{h_{M}}^{E}$ a function with certain form of singularity and applying the least square method to the discrete data of $\mathbf{u}_{h}$ to fit the parameters in the function, the value of $\alpha_{h_{M}}$ is finally taken to be the one which produces the least difference between the discrete data of $\mathbf{u}_{h}$ and the fitted singular function.

4.3. Numerical results. First, we describe the triangulations used in our numerical experiments. Given positive integers $N_{M}$ and $L_{M}$, let $r_{i}^{M}=\frac{i}{N_{M}}(i=$ $\left.0,1, \cdots, N_{M}\right)$ and $\theta_{i, j}^{M}=\frac{j \pi}{i L_{M}}\left(i=0,1, \cdots, N_{M}, j=0,1, \cdots, i L_{M}\right)$. The triangulation points are defined by $\mathbf{x}_{i, j}^{M}=\left(x_{1, i, j}^{M}, x_{2, i, j}^{M}\right)$, where $x_{1, i, j}^{M}=r_{i}^{M} \cos \left(\theta_{i, j}^{M}\right)$ and $x_{2, i, j}^{M}=r_{i}^{M} \sin \left(\theta_{i, j}^{M}\right)\left(i=0,1, \cdots, N_{M}\right.$ and $\left.j=0,1, \cdots, i L_{M}\right)$. Then the triangulation $\mathcal{T}^{h_{M}}$, with $h_{M}$ being the maximum diameter of triangulation units, are obtained by connecting these points as shown in Figure 2, where $N_{M}=5$ and $L_{M}=3$. On such triangulations, in consistent with the admissible deformation defined by (4.3), we define the admissible set of finite element functions

$$
\begin{aligned}
\mathcal{A}_{h_{M}}=\left\{\mathbf{u} \in C\left(\bar{\Omega}_{\pi} ; R^{2}\right)\right. & :\left.\mathbf{u}\right|_{K} \text { is affine, } \forall K \in \mathcal{T}^{h_{M}}, \text { and } \\
& \left.\mathbf{u} \text { satisfies }\left(B C_{\pi, \frac{\pi}{2}}\right) \text { and } \operatorname{det} D \mathbf{u}(\mathbf{x})>0 \text { a.e. } \mathbf{x} \in \Omega_{\pi}\right\} .
\end{aligned}
$$

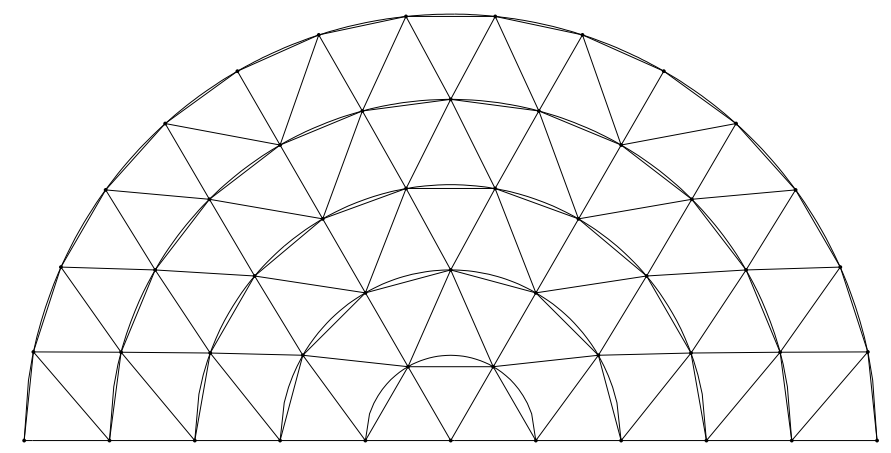

Figure 2. The $5 \times 3$ mesh of $\Omega_{\pi}$, i.e. $N_{M}=5, L_{M}=3$.

Our numerical experiments on $I_{0}$ show that typical numerical solutions obtained by the standard finite element methods have large derivatives near the point $\mathbf{x}_{0,0}^{M}=(0,0)$ and on the line segments $\overline{\mathbf{x}_{1, j}^{M} \mathbf{x}_{1, j+1}^{M}}$ for $j=0,1, \cdots, L_{M}-1$ (see Figure 3 ). Hence the truncation region is initially set to be $\widetilde{\Omega}_{h_{M}}^{E}=\{K \in$ 
$\mathcal{T}^{h_{M}}: \overline{\mathbf{x}_{1, j}^{M} \mathbf{x}_{1, j+1}^{M}} \subset \bar{K}$ or $\left.\mathbf{x}_{0,0}^{M} \in \bar{K}\right\}$. Let $I_{M}^{E}(\mathbf{u} ; p)$ be given by (3.5) and (3.6) with $\alpha_{h_{M}}(\mathbf{x})>\alpha_{1}=10^{-10}$ for all $\mathbf{x} \in \widetilde{\Omega}_{h_{M}}^{E}$. For simplicity, $\alpha_{h_{M}}(\mathbf{x})$ is taken to be constant $\bar{\alpha}_{h_{M}}$ for all $\mathbf{x} \in \widetilde{\Omega}_{h_{M}}^{E}$. After some iterations with the truncation method, the norm of the gradient near the origin $E=\{(0,0)\}$ increases dramatically, while it keeps steady and even drops elsewhere. Our numerical experiments show that, at least in our examples, $h=h_{M}$ is sufficient to guarantee convergence of the algorithm, i.e., there is no need for further mesh refinement as might be expected by the general convergence theory given in Section 3. A post process with further iterations in which the truncation region is adaptively readjusted to $\widetilde{\Omega}_{h_{M}}^{E}=\left\{K \in \mathcal{T}^{h_{M}}: \mathbf{x}_{0,0}^{M} \in K\right\}$ and $\mathbf{u}_{h_{M}}\left(\mathbf{x}_{1, j}\right)\left(j=0,1, \cdots, L_{M}\right)$ are kept fixed, in other words, the function $\mathbf{u}_{h_{M}}$ is kept fixed on the new truncation region $\widetilde{\Omega}_{h_{M}}^{E}$, effectively accelerated the convergence.

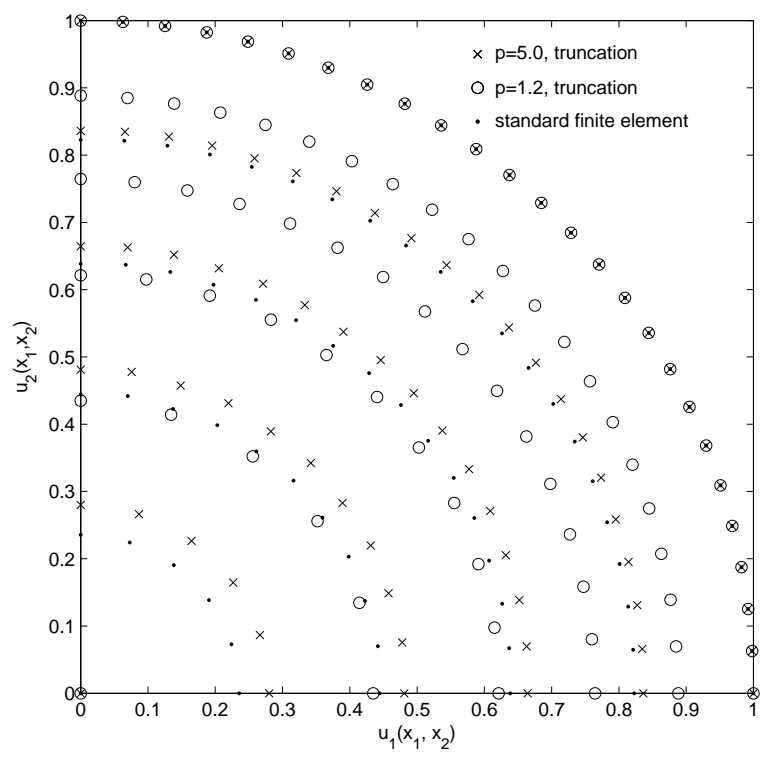

FiguRE 3. The numerical solutions produced by the standard finite element method and the truncation method for $p=1.2,5.0$ with $N_{1}=L_{1}=5$.

The numerical results clearly indicate that the minimizer has a point singularity at the origin. Notice that the nodal values of the numerical solutions $\mathbf{u}_{h_{M}}$ along the $L_{M}+1$ lines in radius directions $\vartheta(\mathbf{x})=\theta_{1, j}^{M}\left(j=0,1, \cdots, L_{M}\right)$ are $\mathbf{u}_{h_{M}}\left(r_{i}^{M} \cos \left(\theta_{i, i \times j}^{M}\right), r_{i}^{M} \sin \left(\theta_{i, i \times j}^{M}\right)\right)$ and $\theta_{i, i \times j}^{M}=\theta_{1, j}^{M}$ for $i=1,2, \cdots, N_{M}$. To get a 
better description of the singularity, we assume that, for each $j=0,1, \cdots, L_{M}$, the leading term of the singularity has the form $\gamma_{\theta_{1, j}^{M}} r(\mathbf{x})^{s_{\theta_{1, j}^{M}}}$, and evaluate $\gamma_{\theta_{1, j}^{M}}>0$ and $s_{\theta_{1, j}^{M}} \in(0,1)$ by the least square method using the values of $\left\{\mathbf{u}_{h_{M}}\left(r_{i}^{M} \cos \left(\theta_{i, i \times j}^{M}\right), r_{i}^{M} \sin \left(\theta_{i, i \times j}^{M}\right)\right)\right\}_{i=1}^{k}$ near the singular set $E=\{(0,0)\}$, and the truncation parameter $\bar{\alpha}_{h_{M}}$ are taken so that the sum of the $l^{2}$-error between $\mathbf{u}_{h_{M}}$ and $\gamma_{\theta_{1, j}^{M}} r(\mathbf{x})^{s_{\theta_{1, j}^{M}}}$ on $\left\{\left(r_{i}^{M} \cos \left(\theta_{i, i \times j}^{M}\right), r_{i}^{M} \sin \left(\theta_{i, i \times j}^{M}\right)\right)\right\}_{i=1}^{k}$ is minimized, where $k \leq N_{M}$ is a given integer. In our numerical experiments we set $k=3$. We notice that the numerical results are not very sensitive to the parameter $\bar{\alpha}_{h_{M}}$.

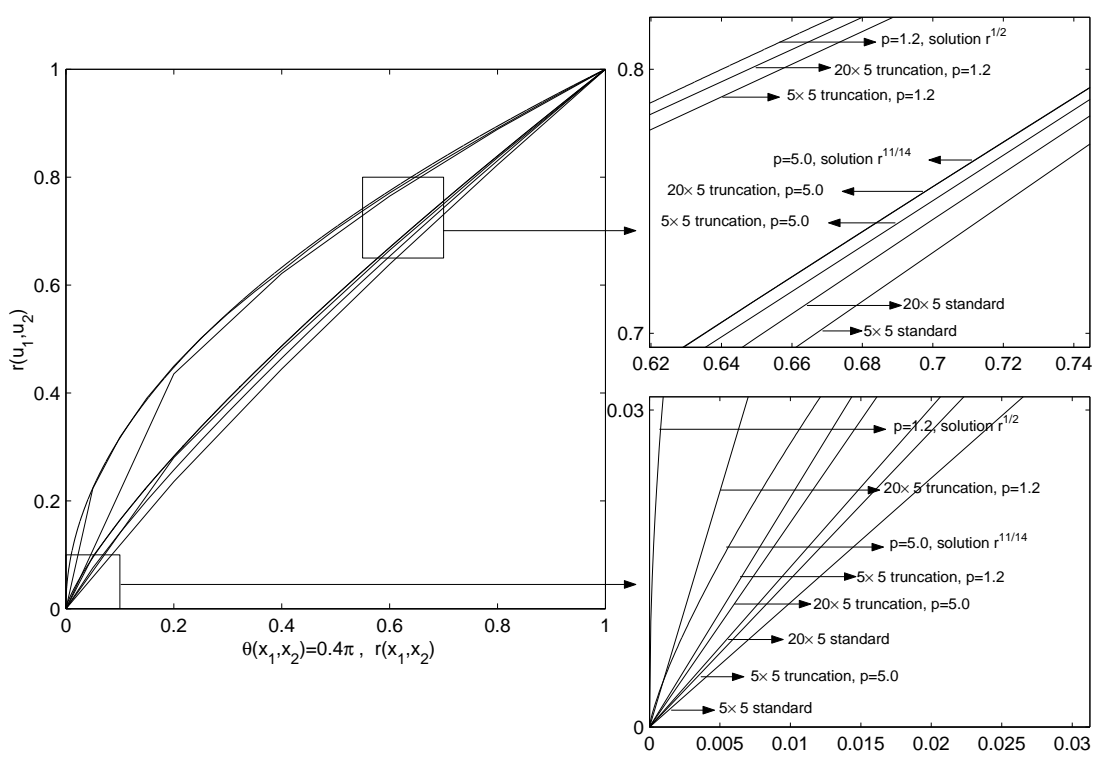

FiguRE 4. The numerical solutions $r\left(\mathbf{u}_{h_{M}}(\mathbf{x})\right)(M=1,3)$ on $\vartheta(\mathbf{x})=0.4 \pi$ for $I_{0}$ and $p=1.2,5.0$.

Some numerical results for $I_{0}$ are shown in Figure 3 - 5. The numerical solutions $\mathbf{u}_{h_{1}}$ produced by the truncation method with $N_{1}=L_{1}=5$ and the optimal truncation parameter $\bar{\alpha}_{h_{1}}=2 \times 10^{-6}$ for $p=1.2, \bar{\alpha}_{h_{1}}=5 \times 10^{-4}$ for $p=5.0$ respectively are shown in Figure 3. For $N_{3}=20$ and $L_{3}=5$, the optimal truncation parameter $\bar{\alpha}_{h_{3}}$ obtained by our numerical experiments are $\bar{\alpha}_{h_{3}}=3 \times 10^{-6}$ for $p=1.2$ and $\bar{\alpha}_{h_{3}}=1.5 \times 10^{-3}$ for $p=5.0$ respectively. The numerical solutions $r\left(\mathbf{u}_{h_{M}}(\mathbf{x})\right)(M=1,3)$, produced by the truncation method and the standard finite element method, on a radius line $\vartheta(\mathbf{x})=0.4 \pi$ are shown in Figure 4, where the Lavrentiev gap in singularity can be easily spotted. The 
convergence behavior of the truncation method for $I_{0}$ with respect to $N_{M}$ for $p=1.2$ and $p=5.0$ is shown in Figure 5. We point out here that the numerical experiments show that the convergence behavior of the algorithm is essentially the same for various $L_{M}$. This is not surprising, since the solution of the problem is linear in $\theta$ in the polar coordinates (see Theorem 4.2).
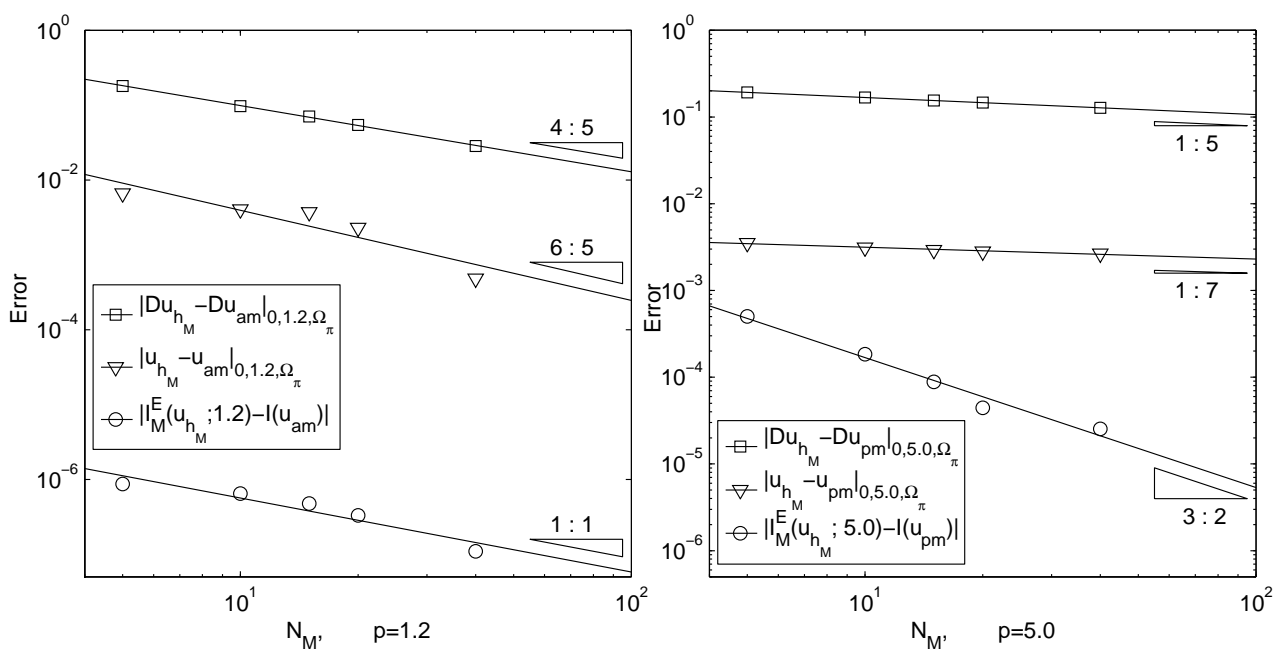

FiguRE 5. Convergence rates of the truncation method for $I_{0}$, $p=1.2$ and $p=5.0$ with the minimizer $\mathbf{u}_{a m}$ and $\mathbf{u}_{p m}$ respectively.

For the case of $I_{\varepsilon, \kappa}$, we take $p=2.2$ and 5.0 respectively, when the stored energy density $W_{\varepsilon, \kappa}$ is polyconvex, and we take $\kappa=3$. Thus, according to the theory of Foss et. al. [13] (see Theorem 4.2), $I_{\varepsilon, 3}$ should exhibit the Lavrentiev phenomenon for $\varepsilon$ satisfying $0<\varepsilon<\varepsilon_{\pi, \frac{\pi}{2}, 3} \approx 3.19137 \times 10^{-5}$. This is verified by our numerical experiments with the truncation method using the same techniques as is described above for the case of $I_{0}$, which suggests that the perturbation upper bound can be improved from $\varepsilon_{\pi, \frac{\pi}{2}, 3}$ to $\varepsilon_{\pi, \frac{\pi}{2}, 3}^{n u m} \approx 0.02$. In Figure 6 the numerical solutions $\mathbf{u}_{h_{1}, \epsilon}\left(N_{1}=L_{1}=5\right)$ for $I_{\epsilon, 3}$ with $\epsilon=10^{-5}$ produced by the truncation method using $\bar{\alpha}_{h_{1}}=2 \times 10^{-6}$ for $p=2.2, \bar{\alpha}_{h_{1}}=5 \times 10^{-4}$ for $p=5.0$ respectively are shown. For $N_{2}=10$ and $L_{2}=5$, the numerical solutions $\mathbf{u}_{h_{2}}=\mathbf{u}_{h_{2}, 0}$ and $\mathbf{u}_{h_{2}, \epsilon}$ with $\epsilon=10^{-7}, 10^{-5}, 10^{-3}$ for $I_{\epsilon, 3}$ are produced by the truncation method using $\bar{\alpha}_{h_{2}}=9 \times 10^{-7}$ for $p=2.2$, and the numerical results of $r\left(\mathbf{u}_{h_{2}, \varepsilon}(\mathbf{x})\right)$ on the radius line $\vartheta(\mathbf{x})=0.4 \pi$ are shown in Figure 7 , where it is clearly seen that 
the Lavrentiev gap increases as the parameter $\varepsilon$ decreases. For $p=5.0$, similar numerical results can be obtained.

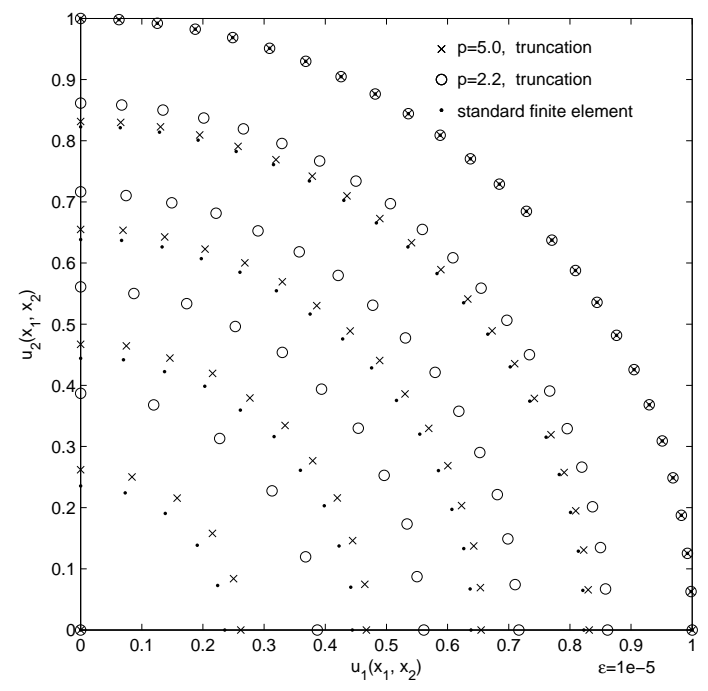

FiguRE 6 . The numerical solution $\mathbf{u}_{h_{1}, 10^{-5}}$ of $I_{10^{-5}, 3}$ produced by the standard finite element method and the truncation method for $p=2.2,5.0$ with $N_{1}=L_{1}=5$.

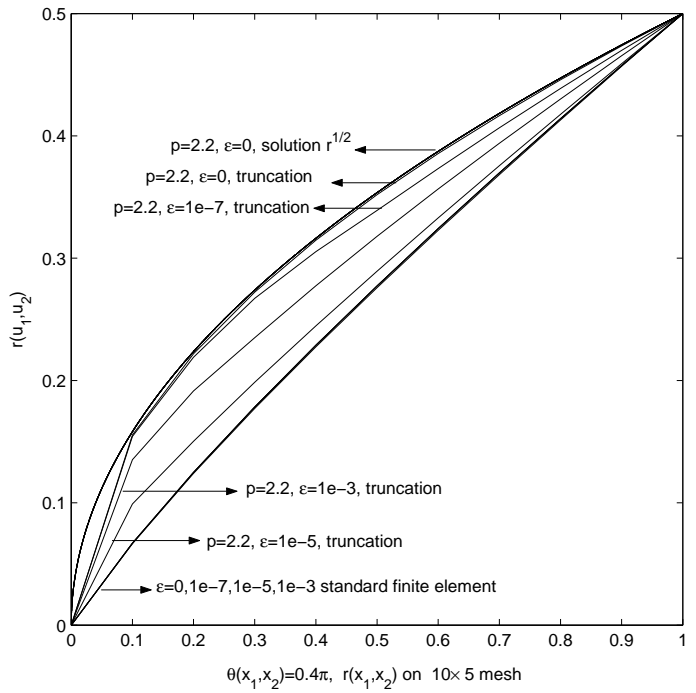

FiguRE 7. The numerical solutions $r\left(\mathbf{u}_{h_{2}, \varepsilon}(\mathbf{x})\right)$ on $\vartheta(\mathbf{x})=0.4 \pi$ for $I_{\epsilon, 3}$ with $\epsilon=0,10^{-7}, 10^{-5}, 10^{-3}$ and $p=2.2$. 
The order of singularity of the numerical solutions $r\left(\mathbf{u}_{h_{M}, \varepsilon}(\mathbf{x})\right)$ can be estimated in the same way as is described for the case of $I_{0}$. The numerical results for $p=2.2$ and 5.0 on the radius line $\vartheta(\mathbf{x})=0.4 \pi$ are shown in Figure 8. Assuming that the numerical order of singularity $s^{\left\{N_{M}\right\}}$ converges to the order of singularity $s$ in such a way that

$$
s^{\left\{N_{M}\right\}} \approx s+a_{1} N_{M}^{-b_{1}}+a_{2} N_{M}^{-b_{2}}
$$

and setting $b_{1}=1.25, b_{2}=2.25$ for $p=2.2$ and $b_{1}=2.5, b_{2}=3.5$ for $p=5.0$, as we found the data is thus fitted reasonably well. We use our numerical results on $s^{\left\{N_{M}\right\}}$ and the least square method to approximately describe the convergence behavior and especially to approximately obtain the singular order $s$ for various $\varepsilon$ and $p=2.2,5.0$. The numerical results thus obtained are shown in Table 1 , where we see that as $\varepsilon \rightarrow 0$ the estimated order of singularity $s$ decreases nicely to about $\frac{1}{2}$ (for $p=2.2$ ) and $\frac{11}{14} \approx 0.7857$ (for $p=5.0$ ) respectively, which are exactly the orders of singularity of $\mathbf{u}_{a m}$ and $\mathbf{u}_{p m}$ (see Theorem 4.2). The numerical values of $\left(I_{\varepsilon, 3}\right)_{2}^{E}$ for various $\varepsilon$ with $E=\{(0,0)\}, p=2.2,5.0$ and $N_{2}=10, L_{2}=5$ are shown in Figure 9, where we see that the Lavrentiev gap exists for $\varepsilon$ satisfying $0<\varepsilon<\varepsilon_{\pi, \frac{\pi}{2}, 3}^{n u m} \approx 0.02$ and is gradually squeezed to a point as $\varepsilon$ approaches $\varepsilon_{\pi, \frac{\pi}{2}, 3}^{n u m}$.
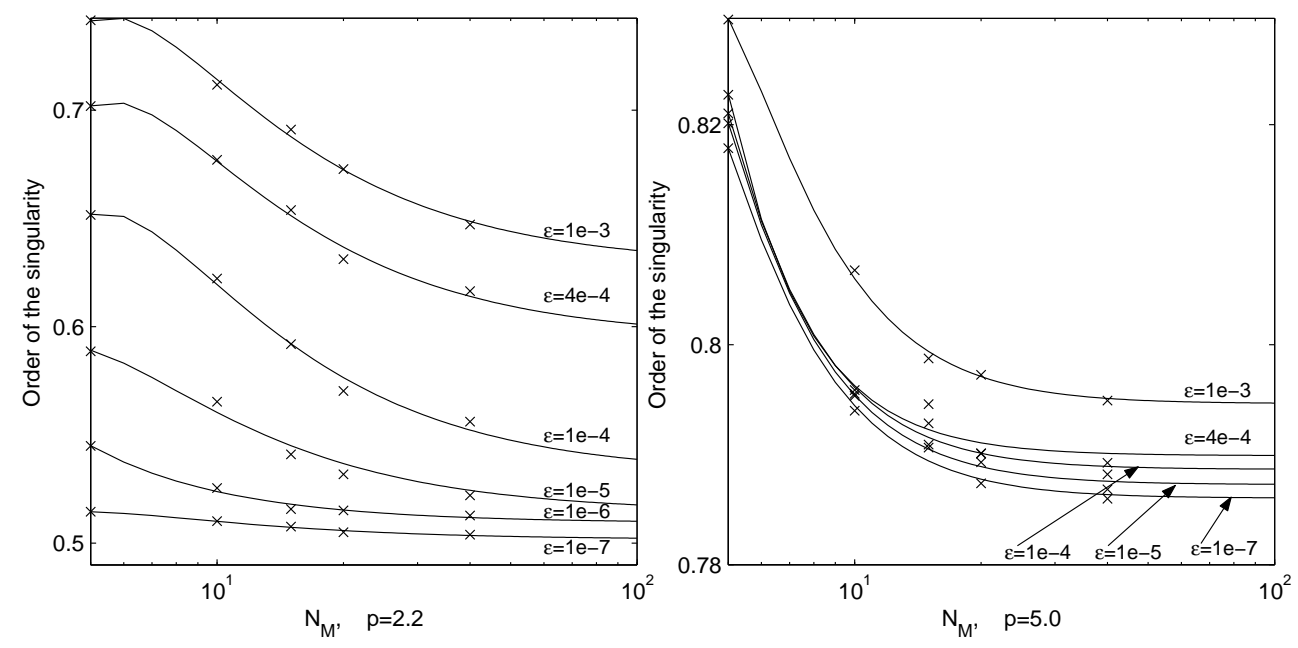

FiguRE 8. The numerical order of singularity of $r\left(\mathbf{u}_{h_{M}, \varepsilon}(\mathbf{x})\right)$ on $\vartheta(\mathbf{x})=0.4 \pi$ for various $\varepsilon$. Left is for $p=2.2$, right for $p=5.0$. 

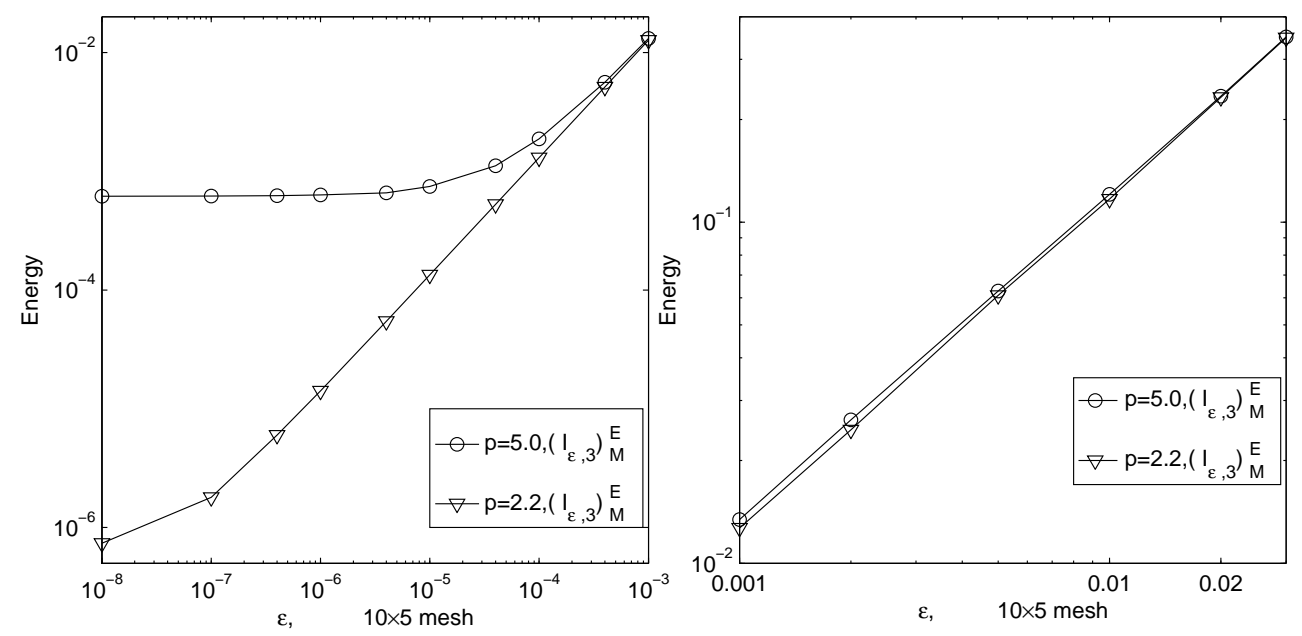

Figure 9. The numerical values of $\left(I_{\epsilon, 3}\right)_{2}^{E}$ for various $\varepsilon$ with $E=\{(0,0)\}, p=2.2,5.0$ and $N_{2}=10, L_{2}=5$.

TABLE 1. The coefficients of the fitting function of the order of singularity of the numerical solutions for $p=2.2,5.0$.

\begin{tabular}{|c|c|c|c|c|c||c|c|c|c|c|}
\hline$\varepsilon$ & \multicolumn{5}{|c||}{$p=2.2$} & \multicolumn{5}{c|}{$p=5.0$} \\
\cline { 2 - 11 } & $s$ & $a_{1}$ & $b_{1}$ & $a_{2}$ & $b_{2}$ & $s$ & $a_{1}$ & $b_{1}$ & $a_{2}$ & $b_{2}$ \\
\hline $10^{-3}$ & 0.6284 & 2.2056 & 1.25 & -6.8068 & 2.25 & 0.7946 & 5.2266 & 2.5 & -16.3448 & 3.5 \\
\hline $10^{-4}$ & 0.5319 & 2.2176 & 1.25 & -6.5984 & 2.25 & 0.7887 & 2.9044 & 2.5 & -5.4808 & 3.5 \\
\hline $10^{-5}$ & 0.5143 & 1.0801 & 1.25 & -2.6018 & 2.25 & 0.7873 & 3.3044 & 2.5 & -7.3331 & 3.5 \\
\hline $10^{-6}$ & 0.5093 & 0.2499 & 1.25 & 0.0861 & 2.25 & 0.7866 & 3.4785 & 2.5 & -8.0505 & 3.5 \\
\hline $10^{-7}$ & 0.5017 & 0.2027 & 1.25 & -0.5331 & 2.25 & 0.7860 & 3.5408 & 2.5 & -8.8179 & 3.5 \\
\hline $10^{-8}$ & 0.4968 & 0.2542 & 1.25 & -0.8198 & 2.25 & 0.7859 & 3.5501 & 2.5 & -8.8235 & 3.5 \\
\hline
\end{tabular}

\section{REFERENCES}

[1] R. A. Adams, Sobolev Spaces, Academic Press, New York, 1975.

[2] Y. Bai and Z.-P. Li, A truncation method for detecting singular minimizers involving the Lavrentiev phenomenon. To appear in Math. Models Methods Appli. Sci.

[3] J. M. Ball, Discontinuous equilibrium solutions and caviation in nonlinear elasticity, Philos. Trans. Roy. Soc. London Ser. A 306(1982), 557-611.

[4] J. M. Ball and V. J. Mizel, Singular minimizers for regular one dimensional problems in the calculus of variations, Bull. Am. Math. Soc. 11(1984), 143-146. 
[5] J. M. Ball and V. J. Mizel, One-dimensional variational problems whose minimizers do not satisfy the Euler-Lagrange equation, Arch. Rational Mech. Anal. 90(1985), 325-388.

[6] J. M. Ball and G. Knowles, A numerical method for detecting singular minimizers, Numer. Math. 51(1987), 181-197.

[7] J. M. Ball, The Calculus of Variations and Material Sciences, Quarterly of Applied Mathematics, 56(1998), 719-740.

[8] P. G. Ciarlet, The Finite Element Method for Elliptic Problems, North HollandAmsterdam, 1978.

[9] B. Dacorogna, Direct Methods in the Calculus of Variations, Apllied Math. Sciences, vol. 78, Springer Verlag, 1989.

[10] A. M. Davie, Singular minimizers in the calculus of variations in one dimension, Arch. Rational Mech. Anal. 101(1988), 161-177.

[11] R. E. Edwards, Functional Analysis, Holt, Rinehart and Winston, 1965.

[12] M. Foss, Examples of the Lavrentiev phenomenon with continuous Sobolev exponent dependence, Journal of Convex Analysis 10(2)(2003), 445-464.

[13] M. Foss, W. Hrusa and V. Mizel, The Lavrentiev gap phenomenon in nonlinear elasticity, Arch. Rational Mech. Anal. 167(2003), 337-365.

[14] S. Lang, Real and Functional Analysis, Springer-Verlag, New York, 1993.

[15] M. Lavrentiev, Sur quelques problèmes du calcul des variations. Ann. Math. Pure Appl. 4(1926), 7-18.

[16] V. Pablo, A numerical method for detecting singular minimizers of multidimensional problems in nonlinear elasticity. Numer. Math. 58(1990), 135-144.

[17] Z.- P. Li, Element removal method for singular minimizers in variational problems involving the Lavrentiev phenomenon, Proc. Roy. Soc. Lond., 439A(1992), 131-137.

[18] Z.-P. Li, A numerical method for computing singular minimizers, Numer. Math. 71(1995), 317-330.

[19] Z.-P. Li, Element removal method for singular minimizers in hyperelasticity, Math. Models Methods Appli. Sci., 5(1995), 387-399.

[20] Z.-P. Li, A theorem on lower semicontinuity of integral functionals, Proc. Roy. Soc. Edinb., 126A(1996), 363-374.

[21] B. Manià, Soppa un esempio di Lavrentieff, Ball. Unione Mat. Ital. 13(1934), 147-153. 